\title{
Non-biological synthetic spike-in controls and the AMPtk software pipeline improve mycobiome data
}

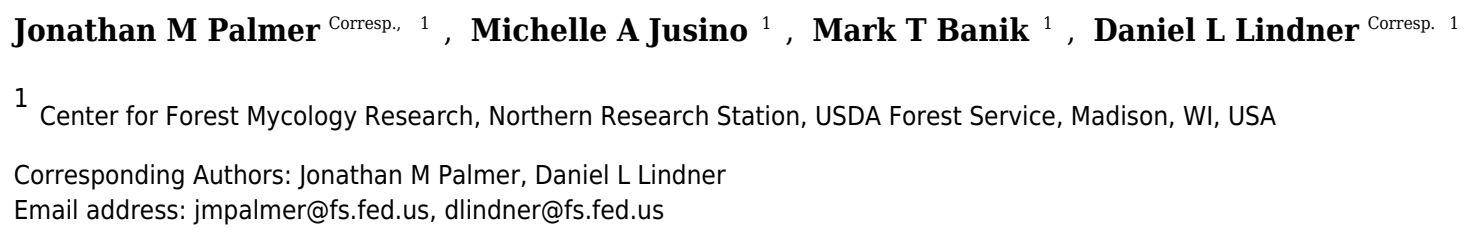

High throughput amplicon sequencing (HTAS) of conserved DNA regions is a powerful technique to characterize microbial communities. Recently, spike-in mock communities have been used to measure accuracy of sequencing platforms and data analysis pipelines.To assess the ability of sequencing platforms and data processing pipelines using fungal ITS amplicons, we created two ITS spike-in control mock communities composed of cloned DNA in plasmids: a biological mock community (BioMock), consisting of ITS sequences from fungal taxa, and a synthetic mock community (SynMock), consisting of non-biological ITS-like sequences. Using these spike-in controls we show that: 1) a nonbiological synthetic control (e.g., SynMock) is the best solution for parameterizing bioinformatics pipelines, 2 ) pre-clustering steps for variable length amplicons are critically important, 3) a major source of bias is attributed to initial PCR reactions and thus HTAS read abundances are typically not representative of starting values. We developed AMPtk, a versatile software solution equipped to deal with variable length amplicons and quality filter HTAS data based on spike-in controls. While we describe herein a non-biological synthetic mock community for ITS sequences, the concept and AMPtk software can be widely applied to any HTAS dataset to improve data quality. 
1 Non-biological synthetic spike-in controls and the AMPtk software pipeline improve mycobiome

2 data

3

4 Jonathan M. Palmer ${ }^{1 *}$, Michelle A. Jusino ${ }^{1 *}$, Mark T. Banik ${ }^{1}$, and Daniel L. Lindner ${ }^{1}$

5

$6{ }^{1}$ Center for Forest Mycology Research, US Forest Service, Madison, WI 53726, USA

7 *authors contributed equally to this manuscript

8

9 Correspondence:

10

11

12

13

14

15

16

17

18

19

20

21

22

Daniel Lindner

One Gifford Pinchot Drive

Madison, WI 53726

(e) dlindner@fs.fed.us

(p) 608-231-9511

Jonathan Palmer

One Gifford Pinchot Drive

Madison, WI 53726

(e) jmpalmer@fs.fed.us

(p) 608-231-9511

23 
24

25

26

27

28

29

30

31

32

33

34

35

36

37

38

39

40

41

42

43

44

45

46

47

48

49

50

51

\section{Abstract}

High throughput amplicon sequencing (HTAS) of conserved DNA regions is a powerful technique to characterize microbial communities. Recently, spike-in mock communities have been used to measure accuracy of sequencing platforms and data analysis pipelines. To assess the ability of sequencing platforms and data processing pipelines using fungal ITS amplicons, we created two ITS spike-in control mock communities composed of cloned DNA in plasmids: a biological mock community (BioMock), consisting of ITS sequences from fungal taxa, and a synthetic mock community (SynMock), consisting of non-biological ITS-like sequences. Using these spike-in controls we show that: 1) a non-biological synthetic control (e.g., SynMock) is the best solution for parameterizing bioinformatics pipelines, 2) pre-clustering steps for variable length amplicons are critically important, 3) a major source of bias is attributed to initial PCR reactions and thus HTAS read abundances are typically not representative of starting values. We developed AMPtk, a versatile software solution equipped to deal with variable length amplicons and quality filter HTAS data based on spike-in controls. While we describe herein a non-biological synthetic mock community for ITS sequences, the concept and AMPtk software can be widely applied to any HTAS dataset to improve data quality.

Availability and Implementation - AMPtk is publically available at https://github.com/nextgenusfs/amptk. All primary data and data analysis done in this manuscript are available via the Open Science Framework (https://osf.io/4xd9r/). The SynMock sequences and the script to produce them are available in the OSF repository ((https://osf.io/4xd9r/) as well as packaged into AMPtk distributions. 


\section{Introduction}

53

54

55

56

57

58

59

60

61

62

63

64

65

66

67

68

69

70

71

72

73

74

75

76

77

78

79

80

81

82

83

84

85

High-throughput amplicon sequencing (HTAS) is a powerful tool that is frequently used for examining community composition of environmental samples. HTAS has proven to be a robust and cost-effective solution due to the ability to multiplex hundreds of samples on a single next-generation sequencing (NGS) run. However, HTAS output from environmental samples requires careful interpretation and appropriate and consistent use of positive and negative controls (Nguyen et al. 2015). One of the major challenges in HTAS is to differentiate sequencing error versus real biological sequence variation. Considerable progress has been made in the last several years via improved quality of sequencing results through manufacturer upgrades to reagents as well as improved quality filtering and "clustering" algorithms. While most algorithm development in HTAS is focused on the prokaryotic microbiome, using the $16 \mathrm{~S}$ subunit of the rRNA array (e.g. QIIME (Caporaso et al. 2010), Mothur (Schloss et al. 2009), UPARSE (Edgar 2013), DADA2 (Callahan et al. 2016)), many of these same tools have been adopted for use with other groups of organisms, such as fungi.

The internal transcribed spacer (ITS) region of the rRNA array has emerged as the molecular barcode for examining fungal communities in environmental samples (Schoch et al. 2012). The ITS region is multi-copy and thus easily amplifiable via PCR even from environmental samples with low quantities of fungal DNA. The ITS region consists of three subunits, ITS1, 5.8S, and ITS2 (Figure 1A), and is generally conserved within fungal species yet possess enough variability to differentiate among species in many taxonomic groups. Because of its widespread use, several public databases are rich with reference fungal ITS sequences (Schoch et al. 2012). However, there are several properties of fungi and the fungal ITS region that are potentially problematic for HTAS that include: i) fungi have variable cell wall properties making DNA extraction efficiency unequal for different taxa and/or cell types (hyphae, fruiting bodies, spores, etc) (Vesty et al. 2017), ii) the number of nuclei per cell is variable between taxa (Roper et al. 2011), iii) the number of copies of the rRNA array are different between taxa and in some cases isolates of the same taxa (Ganley \& Kobayashi 2007), iv) a single isolate can have multiple ITS sequences (intragenomic variability; (Lindner \& Banik 2011; Simon \& Weiss 2008)), v) the ITS region is highly variable in length (Schoch et al. 2014), vi) ITS sequences vary in GC content (Wang et al. 2015), and vii) there are a variable number of homopolymer repeats (Hart Miranda et al. 2015). Additionally, current read lengths of commonly used sequencing platforms (Illumina Miseq currently covers $500 \mathrm{bp}(2 \times 300)$ and lon Torrent is $450 \mathrm{bp}$ ) are not long enough to cover the entire length of the ITS region, which is typically longer than $500 \mathrm{bp}$. However, conserved priming sites exist to amplify either the ITS1 region or the ITS2 region, 
86

87

88

89

90

91

92

93

94

95

96

97

98

99

100

101

102

103

104

105

106

107

108

109

110

111

112

113

114

115

116

117

118

which has been shown to be sufficient for taxonomic identification. While several studies have used the ITS1 region for HTAS, the ITS1 region contains introns in some taxa and thus to avoid potential bias it has been suggested that ITS2 region should be the preferred region for fungi (Taylor et al. 2016). Progress has recently been made using single-molecule DNA sequencing (e.g. PacBio) to assess fungal communities with long read lengths (up to 3000 base pairs), but this has not yet been widely adopted due to cost and technical hurdles (James et al. 2016; Kennedy et al. 2018; Tedersoo et al. 2018)\}.

Sequencing error is a known problem across NGS platforms used for HTAS. To address issues with sequencing error and reliability of results from HTAS, it has become increasingly common practice to use spiked-in "mock" community samples as positive controls for the parameterization and optimization of experimental workflows and data processing. Spike-in mock community controls for fungal ITS have been used (Amend et al. 2010; De Filippis et al. 2017; Nguyen et al. 2015; Taylor et al. 2016; Tonge et al. 2014), and have consisted of fungal genomic DNA (gDNA) extracted from tissue from fruiting bodies, cultures, or spores of a number of taxa which are then (usually) combined in equimolar amounts. Mock communities composed of fungal gDNA from fruiting bodies, spores, and/or hyphae provide a measure of success of extraction, PCR, and sequencing and thus are useful in the HTAS workflow. However, such mock communities are of limited value if used to validate/parameterize data processing workflows due to intrinsic properties of the ITS region mentioned previously (variable copy number, intraspecific variation, variable length, etc.). Therefore, there is a need for fungal ITS spike-in control mock communities that function to validate laboratory experimental design, validate data processing steps, and compare results between sequencing runs and platforms.

HTAS is cost-effective due to the ability to massively multiplex environmental samples on a single sequencing run. This process depends on the attachment of a unique sequence identifier (referred to as a barcode, an index, or a tag, depending on sequencing platform) to each piece of DNA to be sequenced. In recent years, "tag-switching" ("index hopping", "tag jumping", "barcode jumping", "index-bleed", or "barcode switching") has been noted to occur on Roche 454 platforms as well as Illumina platforms (Carlsen et al. 2012; Degnan \& Ochman 2012; Kircher et al. 2011; Philippe et al. 2015; Schnell et al. 2015). Tag-switching can lead to over-estimation of diversity in environmental samples (Philippe et al. 2015; Schnell et al. 2015) and mis-assignment of sequences to samples. It has been noted that spike-in mock communities may be useful to help detect tag-switching, and subsequent filters may be applied for use with the HTAS pipeline of choice (Degnan \& Ochman 2012; Philippe et al. 2015). 
We hypothesized that a mock community composed of cloned fungal ITS sequences (in

120

121

122

123

124

125

126

127

128

129

130

131

132

133

134

135

136

137

138

139

140

141

142

143

144

145

146

147

148

149

150

151

152 plasmids) would circumvent several of the variability issues associated with using mock communities composed of fungal DNA or fungal tissue (variable copy number, intraspecific variation, etc), allowing for a definitive assessment of HTAS for mycobiome studies. Subsequently, we found that current "off-the-shelf" software solutions performed poorly using these fungal ITS community standards and thus developed AMPtk (amplicon toolkit), a versatile software pipeline that improves results from HTAS data. Furthermore, we designed a nonbiological synthetic spike-in mock community consisting of ITS-like sequences (SynMock) that, when coupled with AMPtk, provides a simple method to reduce the effects of tag-switching between multiplexed samples on a HTAS run.

\section{Materials and Methods}

\section{Biological mock community (BioMock)}

To construct the BioMock we selected 26 identified fungal cultures (Supporting Information Table S1) from the Center for Forest Mycology Research (CFMR) culture collection (US Forest Service, Madison, Wisconsin). These cultures were purposefully chosen to represent a taxonomic range of fungal species, including species with known ITS paralogs, species with GC rich ITS regions, a variety of ITS lengths, and species with a variety of homopolymers in the ITS region. To measure the sensitivity of our bioinformatics approach, we also included two ITS sequences from Leptoporus mollis that were cloned from the same culture as an example of intragenomic variation in the fungal ITS region. These two sequences are more than $3 \%$ divergent (95.9\% identical) and thus would typically represent separate operational taxonomic units (OTUs) in a clustering pipeline, despite being from the same fungal isolate. All cultures were grown on cellophane on malt extract agar, and DNA was extracted from pure cultures following (Lindner \& Banik 2008). Following extraction, the genomic DNA was PCR amplified using the fungal ITS specific primers ITS-1F (Gardes \& Bruns 1993) and ITS4 (White et al. 1990). PCR products were then cloned into pGEM-T (Promega, Madison, WI) and Sanger sequenced using the ITS1-F primer following the protocol in (Lindner \& Banik 2011). Sequences were verified via BLAST search and two clones of each isolate were selected and cultured in liquid LB (Luria-Bertani) media and incubated at $37 \mathrm{C}$ for 24 hours. Plasmids were purified from the cultures in LB media using standard alkaline lysis. These plasmids will hereafter be termed "purified plasmids". The purified plasmids were then Sanger sequenced with vector primers T7 and SP6 to verify the insertion of a single copy of the appropriate ITS fragment. Purified plasmid DNA was quantified using a Qubit 2.0 fluorometer and DNA 
153 concentrations were equilibrated to $10 \mathrm{nM}$ using DNA-free molecular grade water. Following

154 equilibration, $5 \mu \mathrm{l}$ of each purified plasmid were combined to make an equimolar "biological

155 mock" community of single-copy purified plasmids (BioMock).

156 PCR has known biases, which are related to different sequence characteristics and are

157 hard to predict in mixed DNA communities of unknown composition. To illustrate the impact of

158 initial PCR bias on the number of reads obtained from each member of a mixed DNA

159 community, we generated individual HTAS-compatible PCR products from each BioMock

160 plasmid which were subsequently mixed (post-PCR) in an equimolar ratio. This was

161 accomplished by PCR amplifying each individual plasmid with the same barcoded primer set.

162 PCR products were purified using E-gel囚 CloneWell ${ }^{\mathrm{TM}} 0.8 \%$ SYBRß Safe agarose gels

163 (ThermoFisher), quantified using a Qubit ${ }^{2} 2.0$ fluorometer, and combined into an equimolar

164 mixture post-amplification. This post-PCR combined mock community can be used to examine

165 sequencing error on NGS platforms and is referred to as BioMock-standards.

166

167

Non-biological synthetic mock community (SynMock)

168

We used the well-annotated ribosomal RNA (rRNA) sequence from Saccharomyces

169

cerevisiae as a starting point to design ITS-like synthetic sequences. The ITS adjacent regions

170

of small subunit (SSU) and large subunit (LSU) of S. cerevisiae were chosen as anchoring

171

points because of the presence of conserved priming sites ITS1/ITS1-F and ITS4. A 5.8S

172 sequence was designed using $S$. cerevisiae as a base but nucleotides were altered so it would

173 be compatible with several primers in the 5.8S region, including ITS2, ITS3, and fITS7. Random

174 sequences were generated with constrained GC content and sequence length for the ITS1 and

175 ITS2 regions. Twelve unique sequences were synthesized (Genescript, New Jersey, USA) and

176 cloned into pUC57 harboring ampicillin resistance. The SynMock sequences and the script to

177 produce them are available in the OSF repository (https://osf.io/4xd9r/) as well as packaged into

178 AMPtk distributions (https://github.com/nextgenusfs/amptk). Each plasmid was purified by

179 alkaline lysis, quantified, and an equimolar mixture was created as a template for HTAS library

180 prep.

181

182

Preparation of HTAS libraries and NGS Sequencing

183

HTAS libraries were generated using a proofreading polymerase, Pfx50 (ThermoFisher),

184 and thermocycler conditions were as follows: initial denaturation of $94^{\circ} \mathrm{C}$ for $3 \mathrm{~min}$, followed by 11 cycles of $\left[94^{\circ} \mathrm{C}\right.$ for $30 \mathrm{sec}, 60^{\circ} \mathrm{C}$ for $30 \mathrm{sec}\left(\mathrm{drop} 0.5^{\circ} \mathrm{C}\right.$ per cycle), $68^{\circ} \mathrm{C}$ for $1 \mathrm{~min}$ ], then 26 cycles of $\left[94^{\circ} \mathrm{C}\right.$ for $30 \mathrm{sec}, 55^{\circ} \mathrm{C}$ for $30 \mathrm{sec}$, and $68^{\circ} \mathrm{C}$ for $\left.1 \mathrm{~min}\right]$, with a final extension of $68^{\circ} \mathrm{C}$ 
187 for 7 minutes. PCR products were cleaned using either E-gel $\circledast$ CloneWell ${ }^{\mathrm{TM}} 0.8 \%$ SYBR® Safe 188 agarose gels (Life Technologies) or Zymo Select-a-size spin columns (Zymo Research). All

189 DNA was quantified using a Qubit ${ }^{\circledR} 2.0$ fluorometer with the high-sensitivity DNA quantification 190 kit (Life Technologies).

191 A single step PCR reaction was used to create lon Torrent compatible sequencing

192 libraries (PCR protocol described above), and primers were designed according to

193 manufacturer's recommendations. Briefly, the forward primer was composed of the lon A 194 adapter sequence, followed by the Ion key signal sequence, a unique Ion Xpress Barcode 195 sequence (10-12 bp), a single base-pair linker (A), followed by the fITS7 primer (Ihrmark et al. 196 2012). The reverse primer was composed of the lon trP1 adapter sequence followed by the 197 conserved ITS4 primer (White et al. 1990). Sequencing on the lon Torrent PGM was done

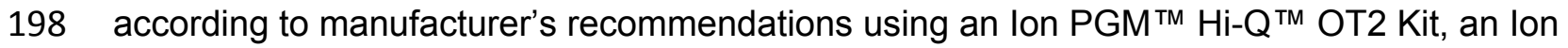
$199 \mathrm{PGM}^{\mathrm{TM}} \mathrm{Hi}-\mathrm{Q}^{\mathrm{TM}}$ Sequencing Kit, an lon $\mathrm{PGM}^{\mathrm{TM}}$ sequencing chip (316v2 or 318v2), and raw data

200

201

202

203

204

205

206

207

208

209

210

211

212

213

214

215

216

217

218

219

220 were processed with the Ion Torrent Suite v5.0.3 with the "--disable-all-filters" flag given to the BaseCaller. Libraries for Illumina MiSeq were generated by a two-step dual indexing strategy. All samples were PCR amplified with Illumina-fITS7 and Illumina-ITS4 primers using PCR protocol described above. PCR products were cleaned and then dual-barcoded using an 8 cycle PCR reaction using the Illumina Nextera Kit and subsequently sequenced using $2 \times 300$ bp sequencing kit on the Illumina MiSeq at the University of Wisconsin Biotechnology Center DNA Sequencing Facility. All primers utilized in this study are available via the OSF repository (https://osf.io/4xd9r/).

Data processing using AMPtk

AMPtk is publically available at https://github.com/nextgenusfs/amptk. All primary data and data analysis done in this manuscript are available via the Open Science Framework (https://osf.io/4xd9r/). AMPtk is written in Python and relies on several modules: edlib (Šošic \& Šikic 2017), biopython (Cock et al. 2009), biom-format (McDonald et al. 2012), pandas (McKinney), numpy (van der Walt et al. 2011), and matplotlib modules (Hunter 2007). External dependencies are USEARCH v9.1.13 (Edgar 2010) or greater and VSEARCH v2.2.0 (Rognes et al. 2016) or greater. In order to run the DADA2 (Callahan et al. 2016) method $R$ is required along with the shortRead (Morgan et al. 2009) and DADA2 packages. The major steps for processing HTAS data are broken down into i) pre-processing reads, ii) clustering into OTUs, iii) filtering OTU table, and iv) assigning taxonomy. 
221 Pre-processing reads - Data structures from Roche 454 and Ion Torrent are similar where

222 reads are in a single file and have a unique barcode at the 5' or 3' end of the read followed by

223 the gene-specific priming site; therefore, AMPtk processes reads from these two platforms very

224 similarly. As a preliminary quality control step, only reads that have a matching barcode

225 sequence (default is to allow 0 mismatches in barcode sequence) and forward primer are

226 retained (default is to allow 2 mismatches in primer sequence). Next, reverse primer sequences

227 are removed and if the amplicons are barcoded on the 3' end the reverse barcode is identified.

228 Finally, the reads are processed in a lossless trimming method that truncates reads longer than

229 the user-defined maximum length but keeps reads shorter than this threshold if they had a valid

230 reverse primer sequence. Data from Illumina is processed differently because reads are most

231 often paired-end reads and most sequencing centers provide users with de-multiplexed by

232 sample paired-end data (i.e. output of 'bcl2fastq'). However, AMPtk also has de-multiplexing

233 methods for the commonly used Earth Microbiome Project format as well as paired-end Illumina

234 data containing barcodes sequences. When processing Illumina data, AMPtk first trims forward

235 and reverser primer sequences, then merges the paired end reads using USEARCH or

236 VSEARCH; phiX spike-in control is removed with USEARCH, and all data are combined into a

237 single file. Pre-processing reads in AMPtk from any of the sequencing platforms results in a

238 single output file that is compatible with all downstream steps.

239

240 Clustering reads into OTUs - AMPtk is capable of running several different clustering algorithms

241 including UPARSE, DADA2, UNOISE2, UNOISE3, and reference-based clustering. The

242 algorithms all start with quality filtering using expected errors trimming and are modified slightly

243 in AMPtk to build OTU tables using the de-multiplexed data (not the quality filtered data) using a

$24497 \%$ percent identity to OTU threshold; therefore read counts represent what was in the sample

245 prior to quality filtering. This is an important distinction, as using variable length amplicons

246 coupled with quality filtering using expected error trimming (Edgar \& Flyvbjerg 2015) can bias

247 longer reads, further distorting read abundance in the final OTU tables.

248

249 Filtering OTU tables to alleviate Tag-switching- Filtering in AMPtk works optimally when a

250 spike-in mock community is sequenced in the dataset. While by default AMPtk is setup to work

251 with the SynMock described herein, any spike-in mock community can be used. AMPtk

252 identifies which OTUs belong to the mock community and calculates tag-switching rate of that

253 mock community into other samples as well as into the mock community from samples. This

254 calculated tag-switching percentage is then used to filter the OTU table. Filtering is done on a 
255

256

257

258

259

260

261

262

263

264

265

266

267

268

269

270

271

272

273

274

275

276

277

278

279

280

281

282

283

284

285

286

287

288

per OTU basis, such that read counts in each OTU that are below the tag-switching threshold are set to zero as they fall within the range of data that could be attributed to tag-switching error and read counts above the threshold are not changed.

Assigning taxonomy - AMPtk is pre-configured with databases for fungal ITS, fungal LSU, arthropod mtCO1, and prokaryotic 16S; however custom databases are easily created with the 'amptk database' command. Several tools are available for taxonomy assignment in AMPtk including remote blast of the NCBI nt database, RDP Classifier (Wang et al. 2007), global alignment to a custom sequence database, UTAX Classifier (RC Edgar, http://drive5.com/usearch/manual9.2/cmd_utax.html), and the SINTAX Classifier (Edgar 2016).

The default method for taxonomy assignment in AMPtk is a "hybrid" approach that uses classification from global alignment, UTAX, and SINTAX. The best taxonomy is then chosen as follows: i) if global alignment percent identity is $>97 \%$ then the top hit is retained, ii) if global alignment percent identity is $<97 \%$, then the best confidence score from UTAX or SINTAX is used, iii) if there is disagreement between taxonomy levels assigned by each method then a least common ancestor (LCA) approach is utilized to generate a conservative estimate of taxonomy. AMPtk also can take a QIIME-like mapping file that can contain all the metadata associated with the HTAS study; the output is then a multi-fasta file containing taxonomy in the headers, a classic OTU table with taxonomy appended, and a BIOM file incorporating the OTU table, taxonomy, and metadata. The BIOM output of AMPtk is compatible with several downstream statistical and visualization software packages such as PhyloSeq (McMurdie \& Holmes 2013) and the vegan package in R (Jari et al. 2017).

Accessory scripts in AMPtk - AMPtk has several additional features that will aid the user in analyzing HTAS data. For instance, AMPtk contains a script that will prepare data for submission to the NCBI SRA archive by formatting it properly and outputting a ready-to-submit SRA submission file. The FunGuild (Nguyen et al. 2016) package which assigns OTUs to an annotated database of functional guilds is also incorporated directly into AMPtk. Additionally, users can draw a heatmap of an OTU table as well as summarize taxonomy in a stacked histogram.

\section{HTAS software pipeline comparison}

For comparison of software pipelines, sequencing data from the lon Torrent PGM and Illumina MiSeq consisted of a single SynMock library, a single BioMock library, and 16 
289

290

291

292

293

294

295

296

297

298

299

300

301

302

303

304

305

306

307

308

309

310

311

312

313

314

315

316

317

318

319

320

321

322

environmental sample libraries. To compare the outputs of these pipelines, OTUs and a corresponding OTU tables were generated using AMPtk v1.2.1, QIIME2 v2018.2, UPARSE (USEARCH v10.0.240), and PIPITS v2.0 (Illumina data only). Read length was set to $300 \mathrm{bp}$ where appropriate for each tool and default or recommended settings were used for each software pipeline with the following exceptions in UPARSE, for processing the Illumina data VSEARCH was used to quality filter, de-replicate (identify unique sequences), and generate an OTU table because the file size was too large to be supported by 32-bit version of USEARCH. QIIME2 workflow utilized the 'qiime cutadapt` module to de-multiplex the lon Torrent PGM data and to remove primers from both datasets. Clustering was done in QIIME2 using DADA2 followed by $97 \%$ clustering using VSEARCH, which is the same methodology used in the 'amptk dada2 ' method. SynMock and BioMock communities were analyzed using 'amptk filter' command for each pipeline to map the OTUs to the corresponding mock communities. Error rate for each mock community was calculated as a percent of the total number of mismatches divided by the total number of base pairs. Scripts used for analysis are available at the Open Science Framework repository (https://osf.io/4xd9r/).

\section{Results}

In silico analysis of the fungal ITS region

To gain baseline data on potential amplicons of the ITS1 or ITS2 regions, the ITS1 and ITS2 regions were extracted with the 'amptk database' command using priming sites specific for each region (ITS1: ITS1-F and ITS2 primer sequences; ITS2: fITS7 and ITS4 primer sequences) from the UNITE+INSD v7.2 database (Abarenkov et al. 2010) consisting of 736,375 ITS sequences. For comparison, the commonly sequenced V3-V4 region was extracted using the 'amptk database' command from prokaryotic $16 \mathrm{~S}$ sequences from the Silva v128 database (Quast et al. 2013). A length histogram for each dataset as well as summary statistics were generated (Figure 1B; Table 1), indicating that all three of these molecular barcodes have an average length of $\sim 250 \mathrm{bp}$ (Table1); however, there was considerable variation in the lengths of the ITS region in comparison to the V3/V4 region of 16S (Figure 1B). Stretches of homopolymer sequences can also be problematic for some NGS platforms (454 and PGM), and thus the number of sequences in this dataset that contained homopolymer stretches greater than 6 nucleotides were calculated using the 'find_homopolymers.py' script in the AMPtk distribution (Table 1). Given the small percentage of ITS1 and ITS2 regions that are greater than $450 \mathrm{bp}$ (the current upper limit of the Ion Torrent PGM platform), the number of taxa in the reference database that are unlikely to sequence on the lon Torrent due to amplicon length is relatively 
323 small (Table 1). Illumina MiSeq is now capable of paired end 300 bp read lengths ( $2 \times 300)$;

324 however, reads need to overlap for proper processing in NGS software platforms and thus a

325500 bp size limit would also be able to sequence most taxa in the reference database using

326 either the ITS1 or ITS2 region.

327

328

\section{Existing data processing workflows perform poorly with fungal ITS sequences}

We cloned known ITS sequences from 26 cultures from the CFMR culture collection that varied in length (237 bp to 548 bp), ranged in GC content (43.8\% - 68.4\%), and contained sequences with homopolymer stretches with one sequence containing two 9 bp stretches. These plasmids were combined into BioMock and BioMock-standards as described in materials and methods section. The value of the BioMock-standards is that the library was combined after PCR, and thus the standards are free from PCR-induced artifacts that may arise from PCR amplification of a mixed community. Clustering amplicons into operational taxonomic units (OTUs) is common practice in molecular ecology and there are many software solutions/algorithms (such as QIIME (Caporaso et al. 2010), UPARSE (Edgar 2013), Mothur (Schloss et al. 2009), and DADA2 (Callahan et al. 2016)) that have been developed to deal appropriately with errors associated with next-generation sequencing platforms. Many studies using 16S amplicon data have focused on comparing clustering methods (Callahan et al. 2016;

341 Edgar 2013), while others have focused on quality filtering reads prior to clustering (Edgar \&

342 Flyvbjerg 2015). At the time these data were generated (2014-2015), there were very few

343 options for pre-processing data from the lon Torrent platform; thus we used the compatible 454

344 method in QIIME v1.9 to de-multiplex the sequencing data (minimum length=100, maximum

345 length $=550$, maximum length homopolymers=10, maximum primer mismatch=2). These data 346 were then clustered using UCLUST, SWARM, and USEARCH in QIIME v1.9 as well as

347 UPARSE (usearch v7.0.1090). However the number of OTUs was highly over-estimated for our 348 mock communities and the error rates were very high (Supporting Information Table S2). The 349 best performing clustering pipeline was UPARSE; however the number of OTUs predicted for 350 the 12 member SynMock was unacceptably high, resulting in 82 OTUs with the lon Torrent 351 PGM data while the 38 OTUs for Illumina MiSeq data was slightly more accurate (Supporting 352 Information Table S2). Based on advances in the UPARSE pipeline and the importance of 353 quality trimming outlined by Edgar \& Flyvbjerg (2015), we speculated that these high error rates 354 and inflated OTU counts could be due to the pre-processing of reads (finding barcodes/primers 355 and quality trimming the sequence data) allowing errors in the data. We were unable to run our 356 data through Mothur due to the inability to do a multiple sequence alignment and subsequent 
357

358

359

360

361

362

363

364

365

366

367

368

369

370

371

372

373

374

375

376

377

378

379

380

381

382

383

384

385

386

387

388

389

390

distance matrix of the ITS region. It is important to note that with the exception of

USEARCH/UPARSE, these software solutions have been built with $16 \mathrm{~S}$ amplicons in mind and several have been optimized for Illumina data.

One major difference in 16S amplicons versus those of ITS1/ITS2 is that the lengths of 16S amplicons are nearly identical, while ITS1/ITS2 amplicons vary in length (Figure 1B). The variable length coupled with sequence divergence of the ITS region between diverse taxa, makes the ITS region impossible to align in many cases (Schoch et al. 2012) and thus represents a major limitation in data processing (i.e. sequence alignment is required for default Mothur OTU clustering). To illustrate the importance of properly pre-processing ITS data, we clustered the ITS1 and ITS2 regions using UPARSE while using the full length ITS1/ITS2 UNITE reference database as a benchmark (Figure 2). Using the UNITE database, we then explored the outcome of trimming/truncating the sequences to different length thresholds, a common practice in OTU clustering pipelines. The UPARSE algorithm uses global alignment and as such terminal mismatches count in the alignment (as opposed to local alignment where terminal mismatches are ignored); thus the UPARSE pipeline expects that reads are truncated to a set length. The original UPARSE algorithm achieves this by truncating all reads to a set length threshold and discards reads that are shorter than the length threshold. Therefore real ITS sequences are discarded (Figure 2). We then came up with two potential solutions to fix this unintended outcome: i) truncate reads that were longer than the threshold and keep all shorter reads (full length), and ii) truncate longer reads and pad the shorter reads with N's out to the length threshold (padding). Using the UNITE v7.2 database of curated sequences (general release June $28^{\text {th }}, 2017$ ) as input, both "full-length" and "padding" improved UPARSE results with the "full length" method recovering more than $99 \%$ of the expected OTUs (Figure 2). It should be noted that the recent version of USEARCH (versions > v8.0.1611) includes a similar method to allow reads to be padded with N's out to a user-defined length threshold (https://www.drive5.com/usearch/manual/cmd fastx truncate.html).

Due to the intrinsic nature of the variable length ITS amplicons, we needed a data processing solution that would be flexible enough to maintain the full length of the reads, trim reads without data loss, prepare sequencing reads for downstream clustering algorithms, handle a large number of sequences, and support all major NGS platforms. Using the mock communities described herein as a means to validate the results of all data processing steps, we wrote a flexible series of scripts for processing Illumina, lon Torrent, as well as Roche 454 data that are packaged into AMPtk (amplicon tool kit). A flow diagram of AMPtk is illustrated in 
391 Figure 3 and a more thorough description of AMPtk is provided in the material and methods

392 section. A manual for AMPtk is available at $\mathrm{http}$ ://amptk.readthedocs.io/en/latest/. After data are

393 pre-processed with AMPtk via a platform specific method, AMPtk then functions as a wrapper

394 for several popular algorithms including UPARSE, DADA2, UNOISE2, and UNOISE3. Data

395 presented in Figure 2 were generated using AMPtk v1.0.1 while the rest of the data were

396 generated using AMPtk v1.2.1.

397

398

399

400

401

402

403

404

405

406

407

408

409

410

411

AMPtk is a fast and accurate HTAS pipeline

Molecular ecology is a rapidly changing field because methods must keep pace with technological advances in DNA sequencing. Therefore, we compared AMPtk to a handful of currently popular HTAS software pipelines: QIIME2 (https://qiime2.org), USEARCH (Edgar 2013), and PIPITS v2.0 (Gweon Hyun et al. 2015). PIPITS is not a universal HTAS pipeline as it only works with paired-end Illumina data; however, we included it because it uses the ITSx software (Bengtsson-Palme et al. 2013) to extract the ITS region. For analysis of the lon Torrent PGM data AMPtk performed the best by recovering all members of the SynMock and BioMock communities, combined with the lowest error rate, the fastest run time (17 minutes), and the most read counts recovered in the OTU table (Table 2). Both USEARCH and QIIME2 resulted in OTUs that contained either primer or adapter sequence contamination, largely due to incomplete removal of the reverse primer sequence (Table 2). A similar trend was found in processing the Illumina MiSeq data, AMPtk recovered all members of the SynMock and BioMock communities, had low error rates, and recovered the most read counts in the OTU table (Table 3). USEARCH was slightly faster than AMPtk (55 minutes versus 67 minutes respectively), however, it had high error rates for the SynMock community (1.047\%) as well as missed a member of the SynMock community (Table 3). The mock community members that were missed by USEARCH or QIIME2 were those that were shorter amplicon sequences, i.e. both pipelines missed mock6 (ITS2 region is $161 \mathrm{bp}$ ). All pipelines produced more OTUs in each mock community sample than were expected, some of these OTUs are a result of tagswitching and a few others are chimeras that slip through the chimera detection algorithms in UPARSE or DADA2. These OTUs can be filtered out of the dataset using the 'amptk filter' command, however, for transparency and to measure the pipeline performance, the raw data is presented (Table 2; Table 3). PIPITS was unable to identify any members of the SynMock

422 community because the non-biological synthetic mock (SynMock) sequences are not identified 423 by ITS $x$ and thus the SynMock cannot be processed by pipelines that use ITS $x$ as a method to 424 pull out the ITS1 or ITS2 regions (Table 3). 
Read abundances do not represent community abundances: PCR introduces bias

Next-generation sequencing platforms are quantitative if the library to be sequenced is

428

429

430

431

432

433

434

435

436

437

438

439

440

441

442

443

444

445

446

447

448

449

450

451

452

453

454

455

456

457

458 unbiased, as is typically the case with RNA-sequencing and whole genome sequencing library prep protocols. However, PCR of mixed communities has long been shown to introduce bias in next-generation sequencing workflows (Aird et al. 2011; Kebschull \& Zador 2015; Pinto \& Raskin 2012) and use of $16 \mathrm{~S}$ mock communities resulted in read abundance bias (Bokulich et al. 2013; Edgar ; Kozich et al. 2013). For HTAS this is an important caveat, as molecular ecologists are interested in diversity metrics of environmental communities as well as their relative abundance. Through the use of mock communities, several studies have pointed out that read abundance from fungal HTAS are not representative of relative biological abundance (Amend et al. 2010; De Filippis et al. 2017). However, it was recently reported that for a fungal ITS mock community of 8 members, abundances were meaningful (Taylor et al. 2016) and correlations between template abundance and read abundance have been previously reported (Ihrmark et al. 2012). Due to disagreements in the literature many studies use abundancebased metrics to analyze HTAS, perhaps due to the unintended consequences of using presence/absence metrics on data that suffer from tag-switching. We reasoned we could investigate this issue using the ITS BioMock artificial community, which would not suffer from bias associated with DNA extraction, ITS copy numbers, and intraspecific variation. We compared the relative read abundances of BioMock-standards to 3 different combinations of BioMock on both the Ion Torrent PGM and Illumina MiSeq platforms (Figure 4). The BioMockstandards consist of an equimolar mixture of 26 PCR products thereby removing the PCR bias from mixed DNA samples, while the BioMock communities consist of an equimolar mixture of 23 single-copy plasmids. These data show that even in an extreme example of an equally mixed community of cloned ITS sequences, read abundance does not represent actual abundance in the mock community (Figure 4). The majority of the bias is introduced at the initial PCR step, as the post-PCR combined BioMock-standards result in a more equal distribution of reads, albeit not a perfect distribution. We also tested PCR conditions, DNA concentrations, and sample reproducibility on the lon Torrent PGM and in all conditions tested we still observed unequal read abundances (Supporting Information Figure S1).

While the bias via PCR is consistent between sequencing platforms, there is no obvious correlation between length of the read, GC content, nor stretches of homopolymers affecting efficient PCR amplification. For example, Wolfiporia dilatophya (mock11) contains no homopolymer stretches larger than 5, has GC distribution of $54.6 \%$, and is near the median in 
459 length, yet it does not PCR amplify well in the BioMock community (Figure 4). These data also 460 show a size limitation in the Ion Torrent PGM workflow, as Wolfiporia cocos (mock26)

461 sequences very poorly due to its long ITS2 region of $548 \mathrm{bp}$ (Figure 4). Three members of the 462 original 26 members of the BioMock community were dropped (mock24, mock25, mock26) due 463 to persistent problems getting them to amplify/sequence in repeated HTAS on the lon Torrent 464 platform (Supporting Information Figure S1). While mock26 (W. cocos) was likely difficult to 465 sequence due to its long size, mock24 and mock25 consistently failed to amplify in the equal 466 mixture of plasmids for unknown reasons.

467 In HTAS experiments, considerable effort is made to try to sequence to an equal depth 468 for each sample. However, in practice this rarely works perfectly and thus a typical HTAS 469 dataset has a $2-4 \mathrm{X}$ range in number of reads per sample. The depth of sequence range for the 470 HTAS runs presented here is within a range of $2 X$ for each run and the smallest number of 471 reads per sample in any of our sequencing runs was nearly 60,000 (Supporting Information 472 Table S5). Unequal sequencing depth has been used as rationale for explaining the lack of 473 correlation between read abundance and actual abundance. Therefore, random subsampling of 474 reads in each sample prior to clustering (also called rarefying) has been widely used in the 475 literature, despite a compelling statistical argument that this method is flawed (McMurdie \& 476 Holmes 2014). Randomly subsampling reads for each sample using our BioMock community 477 yielded nearly identical read abundance biases (Supporting Information Figure S2). Sequencing 478 depth has been shown to be an important variable for HTAS experiments (Smith \& Peay 2014), 479 therefore it has been recommended that a reads per sample cutoff be used with processing 480 481 environmental datasets (while each dataset is different and should be tested empirically, typically we use $5,000-10,000$ as a minimum number of reads per sample).

482

A non-biological synthetic mock community to measure tag-switching among samples

Tag-switching is a phenomenon that has been described on Roche 454 platform (Carlsen et al. 2012) as well as Illumina platforms (Kircher et al. 2012; Wright \& Vetsigian 2016). A consensus on a mechanism of tag-switching during the sequencing run has yet to be reached. Tag-switching is a significant challenge to overcome as sample crossover has the potential to over-estimate diversity and lead to inaccurate representations of microbial communities, especially considering that read abundance is an unreliable proxy for biological abundance (Figure 4). Using our BioMock sequencing results, we also discovered this phenomenon on both Ion Torrent and Illumina platforms. We calculated the rate of tag-switching in our BioMock Ion Torrent sequencing run to be $0.033 \%$ and on Illumina MiSeq between 
$493 \quad 0.233 \%$ and $0.264 \%$. We also confirmed that tag-switching was happening on the Illumina flow-

494 cell by re-sequencing a subset of Illumina libraries that had shown high tag-switching on the first

495 MiSeq flowcell that did not contain the BioMock (Supporting Information Figure S3). One

496 problem that we noticed in measuring tag-switching using a mock community of actual ITS

497 sequences (BioMock) was that these same taxa in the mock community could be present in

498 environmental samples, which would lead to inaccurate estimation of tag-switching. In our

499 environmental data, it was likely that at least one of the BioMock members was present in

500 several of the environmental samples, suggesting the calculated tag-switching could be over-

501 estimated. To overcome this problem, we designed a non-biological synthetic mock community

502 composed of ITS-like sequences that contained conserved priming sites (SSU and LSU

503 regions), ITS1 region, 5.8S region, and an ITS2 region (Figure 5). We designed the ITS1 and

504 ITS2 portions of the sequences to be non-biological; that is, no similar sequences are known to

505 occur in nature (based on searches of known databases and based on the infinitesimally low

506 probability that a randomly generated sequence would match something found in nature) and

507 therefore these non-biological sequences can be used to accurately track tag-switching in HTAS

508 studies. Using the summary statistics from the analysis of the UNITE reference database for

509 guidance, we also varied the length, GC content, and homopolymer stretches to be

510 representative of real fungal ITS sequences.

511 The SynMock was tested as a spike-in control on both the lon Torrent and Illumina

512 MiSeq platforms. The raw data were processed using AMPtk and clustered using UPARSE.

513 These data illustrate that the synthetic sequences are able to be processed simultaneously with

514 real ITS sequences and provide a way to track the level of tag-switching between multiplexed

515 samples (Figure 6). The increased benefit of being able to track the SynMock sequences as

516 they "bleed" out of the sample allows for a more accurate measurement of tag-switching. Using

517 default Illumina de-multiplexing (allowing 1 mismatch in the index sequence), tag-switching

518 using the SynMock community was $0.057 \%$ (Figure 6C). To determine if allowing mismatches in

519 the index reads was increasing tag-switching, we reprocessed the data with 0 mismatches and

520 found that tag-switching was reduced to $0.036 \%$. While tag-switching was reduced by nearly

521 half, the tradeoff was that 0 mismatches resulted in approximately $10 \%$ fewer reads. For most

522 datasets, a loss of $10 \%$ of the sequencing reads should not be problematic, especially if the

523 benefit is to reduce sample mis-assignment in the data. We noted that in our Illumina dual-

524 indexing library prep that there was increased tag-switching on samples that had a shared

525 reverse index (i7), suggesting that errors are increased at later stages of an Illumina sequencing

526 run (Figure 6B). A similar pattern of increased tag-switching correlating with relaxed primer 
527 mismatch settings was observed with lon Torrent PGM data, although not as drastic. Allowing 1

528 mismatch in the barcode resulted in $0.167 \%$ tag-switching while allowing 0 mismatches in the

529 barcode resulted in $0.156 \%$ tag-switching (Figure $6 \mathrm{C}$ ). While these data would suggest that tag-

530 switching is perhaps higher in lon Torrent PGM datasets, we have subsequently used the

531 SynMock on more than 10 different HTAS Ion Torrent PGM experiments and have since seen

532 much lower levels of tag-switching, occasionally approaching zero.

533 Many environmental samples can contain hundreds of taxa and thus a legitimate

534 concern is that the 12-member SynMock community does not represent a realistic community in

535 terms of diversity in a sample. To test if the SynMock was able to be recovered in a more

536 complex community, we mixed SynMock together with two environmental samples that had

537 more than 200 OTUs in previous sequencing runs. These mixed samples show that SynMock

538 could be recovered from a complex community and the sequences behave like real ITS

539 sequences (Figure 6A). While many studies have set a read count threshold to filter "noisy" data

540 from OTU tables, this threshold has been typically selected arbitrarily, i.e. OTUs with read

541 counts less than 100 or less than $10 \%$ of the total, etc. Use of the SynMock spike-in control

542 allowed for data-driven thresholds to be measured and moreover for the ability to filter the OTU

543 table based on the calculated tag-switching. The AMPtk filter command calculates tag-switching

544 by mapping the OTUs to the mock community and then provides a way to filter the OTU table

545 based on this calculated value. AMPtk filters across each OTU in the table such that if a read

546 abundance value for a particular sample is below the calculated tag-switching threshold it is

547 converted to zero, and therefore difficult to sequence or "low abundance" OTUs are not

548 indiscriminately dropped. Taken together, these data illustrate the utility of a non-biological

549 synthetic mock community in parameterizing data processing steps and importantly providing a

550 method in AMPtk to reduce tag-switching from HTAS datasets. AMPtk provides an easy to use

551 method to accurately process variable length amplicons, cluster them into OTUs or denoise

552 sequences, generate an OTU table, filter the OTU table for tag-switching, and assign taxonomy.

553

554

555

556

\section{Discussion}

Many HTAS studies have the goal of measuring and comparing biological diversity in environmental samples; however, there are technical limitations that need to be understood in

557 order to reach justifiable conclusions. Mock communities and negative controls have been

558 shown to have great utility for HTAS studies, and expanding upon this concept, we present a

559 non-biological synthetic mock community of ITS-like sequences for use as a technical spike-in

560 control for fungal biodiversity studies. Additionally, we describe AMPtk, a software tool kit for 
561 analyzing variable length amplicons such as the fungal ITS1 or ITS2 molecular barcodes. These

562 two tools can be coupled together to validate data processing pipelines and reduce tag-

563 switching from OTU tables prior to downstream community ecology analyses. The concept of a

564 non-biological synthetic spike-in control can be expanded to many different genes and

565 organisms, as was recently described for 16S for microbiome studies (Tourlousse et al. 2017).

566 The ITS region is widely used as a molecular barcode in fungal biodiversity studies as it

567 is easy to amplify and public reference databases are robust. However, HTAS with the ITS

568 region presents some unique challenges due to variability in sequence characteristics such as

569 length and copy number. Most HTAS software development and optimization has been focused

570 on the $16 \mathrm{~S}$ molecular barcode, a region that is near uniform in length across prokaryotic taxa.

571 Thus, there is a need for a software solution that can more accurately account for variable

572 length amplicons. We developed a single-copy mock community based on cloned ITS

573 sequences as a tool to validate and compare different NGS platforms and data processing

574 pipelines. Using an artificial single-copy mock community of cloned ITS sequences in plasmids

575 (BioMock), we determined that the core clustering/denoising algorithms work for variable length

576 amplicons; however, pre-processing techniques widely used for uniform length amplicons

577 introduce significant error into the pipelines. Simplifying the pre-processing of sequencing reads

578 (i.e., identifying unique sequence barcodes, forward/reverse primers, and trimming reads to a

579 uniform length without data loss) resulted in large improvement in downstream OTU clustering.

580 The pre-processing of reads prior to quality filtering is critical for variable length amplicons and

581 is implemented in AMPtk. It should be noted that we originally developed AMPtk several years

582 ago to address a need in pre-processing HTAS data from the lon Torrent platform, more recent

583 versions of USEARCH have taken a similar approach for pre-processing reads allowing for the

584 ability to pad reads with N's. However, there is no documented method to remove reverse

585 primers using USEARCH (v10.0.240).

$586 \quad$ Proper pre-processing of variable length amplicons improves clustering results

587 substantially. However, the BioMock results illustrated that read abundances obtained from

588 HTAS are not a reliable proxy for inferring biological abundance, demonstrating additional

589 assays such as qPCR or metagenomics are required to capture biological abundance. Previous

590 studies have tried to correct for relative abundance by normalization to rRNA copy numbers in

591 16S studies with minimal success (Edgar; Kembel et al. 2012). However this is not something

592 that is currently feasible for fungal HTAS due to lack of knowledge in rRNA copy numbers. It has

593 also been suggested that data transformations (log, square-root, etc) can be used to reduce the

594 effects of abundance bias prior to downstream ecological analysis (Nguyen et al. 2015). While 
595 the data presented here does not support using read abundance as a proxy for biological 596 abundance in HTAS studies, it does support use of presence/absence (binary) metrics as we 597 were able to recover all members of our mock community, even when they were spiked into a 598 diverse environmental sample. We identified the initial PCR reaction (library construction) as the 599 major source of read number bias, a conclusion consistent with the literature (Jusino et al. 2017; 600 Polz \& Cavanaugh 1998; Wu et al. 2010). To reduce PCR artifacts for any assay it is generally 601 accepted that one should use the fewest cycles possible, avoid samples with low DNA quantity, 602 and to use a proofreading polymerase (Oliver et al. 2015). We have tested DNA concentration 603 and PCR cycle numbers for HTAS library generation and subsequent sequencing on the lon 604 Torrent PGM platform, and our results were consistent with these general guidelines 605 (Supporting Information Figure S1). However, following these guidelines is not sufficient to 606 reduce the bias in read abundance from a mixed community from PCR. The lon Torrent PGM 607 platform currently has an amplicon size limit of $\sim 450 \mathrm{bp}$, and thus some very large ITS 608 sequences are difficult to sequence. However, there are only a small number of known ITS1 or 609 ITS2 sequences that are longer than $450 \mathrm{bp}$ (Table 1) and therefore either platform, lon Torrent 610 or MiSeq, provided similar results under the conditions tested.

611 Tag-switching has recently been acknowledged by Illumina (https://tinyurl.com/illumina-

612 hopping), although they limit their acknowledgement to a new flow cell on the HiSeq and

613 NovaSeq platforms. Several studies have shown that older instruments/flowcells have also

614 shown tag-switching, albeit at a much lower rate (Kircher et al. 2012; Wright \& Vetsigian 2016)

615 and tag-switching has been identified on Roche 454 (Carlsen et al. 2012). A dual-indexing 616 approach has been suggested to minimize the tag-switching in datasets (Kozich et al. 2013), 617 however the dual indexing strategy we used in this manuscript was not sufficient to eliminate 618 tag-switching. Tag-switching was much higher if samples shared one of the two index 619 sequences (Figure 6B), suggesting that dual indexing using unique sequences for every sample 620 should further reduce the effects of tag-switching on the lllumina platform. Here we report a low 621 rate of tag-switching on both Ion Torrent and Illumina MiSeq platforms. While the effective rate 622 of tag-switching is low $(<0.2 \%)$, coupled with the fact that read number is not a reliable proxy of 623 community abundance, tag-switching in datasets being analyzed by presence-absence metrics 624 is a problematic scenario. To identify and combat tag-switching, we created a non-biological 625 synthetic mock community (SynMock) of ITS-like sequences that behave like real ITS 626 sequences during the HTAS workflow. Because the SynMock sequences are not known to 627 occur in nature, they can be effectively used to measure tag-switching in a sequencing run. 628 


\section{Conclusions}

630 We propose that HTAS studies of fungal ITS communities can be improved by

631 employing SynMock or a similar non-biological synthetic mock community as a technical control.

632 Additional controls such as a biological mock community of mixed fruiting bodies, spores,

633 hyphae, etc. of taxa of interest are also useful if the experiment is designed to identify the

634 prevalence of particular taxa. The bioinformatics pipeline presented here, AMPtk, was

635 developed to specifically address the quality issues that we have identified by using spike-in

636 mock communities and to provide the scientific community with a necessary tool to study fungal

637 community diversity. AMPtk is a flexible solution that can be used to study other regions used in

638 HTAS, such as mitochondrial cytochrome oxidase 1 (mtCO1) of insects and the large subunit

639 (LSU) of the rRNA array. The goal of AMPtk is to reduce data processing to a few simple steps

640 and to improve the output of HTAS studies. Due to the inherent properties of HTAS and the ITS

641 molecular barcode, we take the position that studies of this nature should be used as a

642 preliminary survey of which taxa present in an ecosystem and that inferring relative abundance

643 from read numbers should be avoided. To understand relative abundance of particular taxa in a

644 community, using the sequence data from HTAS studies coupled with additional independent

645 assays such as taxa specific qPCR or digital PCR would allow for relative abundance

646 determinations. However as DNA sequencing continues to become more cost effective, relative

647 abundance in fungal community ecology experiments can be obtained using metagenomics.

648

649 Acknowledgements

650 We sincerely thank Rita Rentmeester for assisting with the growth of some the cultures used to 651 create the biological mock community.

652

653 Data availability

654 Raw sequencing reads and data processing scripts are available at the Open Science

655 Framework at https://osf.io/4xd9r/. Sequencing data is also available at NCBI Small Read

656 Archive under the SRP144513 accession under the BioProject PRJNA305924.

657

658 
659

660

661

662

663

664

665

666

667

668

669

670

671

672

673

674

675

676

677

678

679

680

681

682

683

684

685

686

687

688

689

690

691

692

693

694

695

696

697

698

699

700

701

702

703

704

705

706

707

708

709

\section{References}

Abarenkov K, Henrik Nilsson R, Larsson K-H, Alexander IJ, Eberhardt U, Erland S, Høiland K, Kjøller R, Larsson E, Pennanen T, Sen R, Taylor AFS, Tedersoo L, Ursing BM, Vrålstad T, Liimatainen K, Peintner U, and Kõljalg U. 2010. The UNITE database for molecular identification of fungi--recent updates and future perspectives. The New phytologist 186:281-285. 10.1111/j.1469-8137.2009.03160.x

Aird D, Ross MG, Chen W-S, Danielsson M, Fennell T, Russ C, Jaffe DB, Nusbaum C, and Gnirke A. 2011. Analyzing and minimizing PCR amplification bias in Illumina sequencing libraries. Genome Biology 12:R18. 10.1186/gb-2011-12-2-r18

Amend AS, Seifert KA, and Bruns TD. 2010. Quantifying microbial communities with 454 pyrosequencing: does read abundance count? Molecular Ecology 19:5555-5565. 10.1111/j.1365-294X.2010.04898.x

Bengtsson-Palme J, Ryberg M, Hartmann M, Branco S, Wang Z, Godhe A, Wit P, Sánchez-García M, Ebersberger I, Sousa F, Amend A, Jumpponen A, Unterseher M, Kristiansson E, Abarenkov K, Bertrand Yann JK, Sanli K, Eriksson KM, Vik U, Veldre V, and Nilsson RH. 2013. Improved software detection and extraction of ITS1 and ITS2 from ribosomal ITS sequences of fungi and other eukaryotes for analysis of environmental sequencing data. Methods in Ecology and Evolution 4:914-919. 10.1111/2041-210X.12073

Bokulich NA, Subramanian S, Faith JJ, Gevers D, Gordon JI, Knight R, Mills DA, and Caporaso JG. 2013. Quality-filtering vastly improves diversity estimates from Illumina amplicon sequencing. Nature Methods 10:57-59. 10.1038/nmeth.2276

Callahan BJ, McMurdie PJ, Rosen MJ, Han AW, Johnson AJA, and Holmes SP. 2016. DADA2: High-resolution sample inference from Illumina amplicon data. Nature Methods.

Caporaso JG, Kuczynski J, Stombaugh J, Bittinger K, Bushman FD, Costello EK, Fierer N, Peña AG, Goodrich JK, Gordon JI, Huttley GA, Kelley ST, Knights D, Koenig JE, Ley RE, Lozupone CA, McDonald D, Muegge BD, Pirrung M, Reeder J, Sevinsky JR, Turnbaugh PJ, Walters WA, Widmann J, Yatsunenko T, Zaneveld J, and Knight R. 2010. QIIME allows analysis of high-throughput community sequencing data. Nature Methods 7:335-336. 10.1038/nmeth.f.303

Carlsen T, Aas AB, Lindner D, Vrålstad T, Schumacher T, and Kauserud H. 2012. Don't make a mista(g)ke: is tag switching an overlooked source of error in amplicon pyrosequencing studies? Fungal Ecology 5:747-749. 10.1016/j.funeco.2012.06.003

Cock PJA, Antao T, Chang JT, Chapman BA, Cox CJ, Dalke A, Friedberg I, Hamelryck T, Kauff F, Wilczynski B, and de Hoon MJL. 2009. Biopython: freely available Python tools for computational molecular biology and bioinformatics. Bioinformatics (Oxford, England) 25:1422-1423. 10.1093/bioinformatics/btp163

De Filippis F, Laiola M, Blaiotta G, and Ercolini D. 2017. Different amplicon targets for sequencing-based studies of fungal diversity. Applied and Environmental Microbiology. 10.1128/AEM.00905-17

Degnan $\mathrm{PH}$, and Ochman H. 2012. Illumina-based analysis of microbial community diversity. The ISME journal 6:183-194.

Edgar R. 2016. SINTAX: a simple non-Bayesian taxonomy classifier for $16 S$ and ITS sequences. bioRxiv:074161.

Edgar RC. UNBIAS: An attempt to correct abundance bias in $16 \mathrm{~S}$ sequencing, with limited success. $10.1101 / 124149$

Edgar RC. 2010. Search and clustering orders of magnitude faster than BLAST. Bioinformatics 26:2460-2461. 10.1093/bioinformatics/btq461

Edgar RC. 2013. UPARSE: highly accurate OTU sequences from microbial amplicon reads. Nature Methods 10:996-998. 10.1038/nmeth.2604 
710

711

712

713

714

715

716

717

718

719

720

721

722

723

724

725

726

727

728

729

730

731

732

733

734

735

736

737

738

739

740

741

742

743

744

745

746

747

748

749

750

751

752

753

754

755

756

757

758

759

760

Edgar RC, and Flyvbjerg H. 2015. Error filtering, pair assembly and error correction for nextgeneration sequencing reads. Bioinformatics. 10.1093/bioinformatics/btv401

Ganley ARD, and Kobayashi T. 2007. Highly efficient concerted evolution in the ribosomal DNA repeats: total rDNA repeat variation revealed by whole-genome shotgun sequence data. Genome Research 17:184-191. 10.1101/gr.5457707

Gardes M, and Bruns T. 1993. ITS primers with enhanced specificity for basidiomycetes application to the identification of mycorrhizae and rusts. Molecular Ecology 2:113-118.

Gweon Hyun S, Oliver A, Taylor J, Booth T, Gibbs M, Read Daniel S, Griffiths Robert I, and Schonrogge K. 2015. PIPITS: an automated pipeline for analyses of fungal internal transcribed spacer sequences from the Illumina sequencing platform. Methods in Ecology and Evolution 6:973-980. 10.1111/2041-210X.12399

Hart Miranda M, Aleklett K, Chagnon PL, Egan C, Ghignone S, Helgason T, Lekberg Y, Öpik M, Pickles Brian J, and Waller L. 2015. Navigating the labyrinth: a guide to sequence-based, community ecology of arbuscular mycorrhizal fungi. New Phytologist 207:235-247. 10.1111/nph.13340

Hunter JD. 2007. Matplotlib: A 2D Graphics Environment. Computing in Science \& Engineering 9:90-95. 10.1109/MCSE.2007.55

Ihrmark K, Bödeker IT, Cruz-Martinez K, Friberg H, Kubartova A, Schenck J, Strid Y, Stenlid J, Brandström-Durling M, and Clemmensen KE. 2012. New primers to amplify the fungal ITS2 region-evaluation by 454-sequencing of artificial and natural communities. FEMS Microbiology Ecology 82:666-677.

James TY, Marino JA, Perfecto I, and Vandermeer J. 2016. Identification of putative coffee rust mycoparasites via single-molecule DNA sequencing of infected pustules. Applied and Environmental Microbiology 82:631-639. 10.1128/AEM.02639-15

Jari O, Blanchet FG, Michael F, Roeland K, Pierre L, Dan M, Peter RM, Hara RBO, Gavin LS, Peter S, Stevens MHH, Eduard S, and Helene W. 2017. vegan: Community Ecology Package.

Jusino M, Banik M, Palmer J, Wray A, Xiao L, Pelton E, Barber J, Kawahara A, Gratton C, Peery M, and Lindner D. 2017. An improved method for utilizing high-throughput amplicon sequencing to determine the diets of insectivorous animals. PeerJ Preprints 5:e3184v3181. 10.7287/peerj.preprints.3184v1

Kebschull JM, and Zador AM. 2015. Sources of PCR-induced distortions in high-throughput sequencing data sets. Nucleic Acids Research 43:e143. 10.1093/nar/gkv717

Kembel SW, Wu M, Eisen JA, and Green JL. 2012. Incorporating 16S gene copy number information improves estimates of microbial diversity and abundance. PLoS Computational Biology 8:e1002743. 10.1371/journal.pcbi.1002743

Kennedy PG, Cline LC, and Song Z. 2018. Probing promise versus performance in longer read fungal metabarcoding. The New phytologist 217:973-976. 10.1111/nph.14883

Kircher M, Heyn P, and Kelso J. 2011. Addressing challenges in the production and analysis of illumina sequencing data. BMC Genomics 12:382. 10.1186/1471-2164-12-382

Kircher M, Sawyer S, and Meyer M. 2012. Double indexing overcomes inaccuracies in multiplex sequencing on the Illumina platform. Nucleic Acids Research 40:e3. 10.1093/nar/gkr771

Kozich JJ, Westcott SL, Baxter NT, Highlander SK, and Schloss PD. 2013. Development of a dual-index sequencing strategy and curation pipeline for analyzing amplicon sequence data on the MiSeq Illumina sequencing platform. Applied and Environmental Microbiology 79:5112-5120. 10.1128/AEM.01043-13

Lindner DL, and Banik MT. 2008. Molecular phylogeny of Laetiporus and other brown rot polypore genera in North America. Mycologia 100:417-430.

Lindner DL, and Banik MT. 2011. Intragenomic variation in the ITS rDNA region obscures phylogenetic relationships and inflates estimates of operational taxonomic units in genus Laetiporus. Mycologia 103:731-740.

Peer] reviewing PDF | (2018:02:25077:1:1:NEW 14 May 2018) 
761

762

763

764

765

766

767

768

769

770

771

772

773

774

775

776

777

778

779

780

781

782

783

784

785

786

787

788

789

790

791

792

793

794

795

796

797

798

799

800

801

802

803

804

805

806

807

808

809

810

811

McDonald D, Clemente JC, Kuczynski J, Rideout JR, Stombaugh J, Wendel D, Wilke A, Huse S, Hufnagle J, Meyer F, Knight R, and Caporaso JG. 2012. The Biological Observation Matrix (BIOM) format or: how I learned to stop worrying and love the ome-ome. Gigascience 1:7. 10.1186/2047-217X-1-7

McKinney W. Data structures for statistical computing in Python. Proceedings of the 9th Python in Science Conference. p 51-56.

McMurdie PJ, and Holmes S. 2013. phyloseq: an R package for reproducible interactive analysis and graphics of microbiome census data. PloS One 8:e61217. 10.1371/journal.pone.0061217

McMurdie PJ, and Holmes S. 2014. Waste not, want not: why rarefying microbiome data is inadmissible. PLoS Computational Biology 10:e1003531. 10.1371/journal.pcbi.1003531

Morgan M, Anders S, Lawrence M, Aboyoun P, Pagès H, and Gentleman R. 2009. ShortRead: a bioconductor package for input, quality assessment and exploration of high-throughput sequence data. Bioinformatics (Oxford, England) 25:2607-2608.

10.1093/bioinformatics/btp450

Nguyen NH, Smith D, Peay K, and Kennedy P. 2015. Parsing ecological signal from noise in next generation amplicon sequencing. The New phytologist 205:1389-1393. 10.1111/nph.12923

Nguyen NH, Song Z, Bates ST, Branco S, and Tedersoo L. 2016. FUNGuild: An open annotation tool for parsing fungal community datasets by ecological guild ScienceDirect. Fungal Ecology.

Oliver AK, Brown SP, Callaham MA, and Jumpponen A. 2015. Polymerase matters: nonproofreading enzymes inflate fungal community richness estimates by up to $15 \%$. Fungal Ecology.

Philippe E, Franck L, and Jan P. 2015. Accurate multiplexing and filtering for high-throughput amplicon-sequencing. Nucleic Acids Research:gkv107.

Pinto AJ, and Raskin L. 2012. PCR biases distort bacterial and archaeal community structure in pyrosequencing datasets. PloS One 7:e43093. 10.1371/journal.pone.0043093

Polz MF, and Cavanaugh CM. 1998. Bias in template-to-product ratios in multitemplate PCR. Applied and Environmental Microbiology 64:3724-3730.

Quast C, Pruesse E, Yilmaz P, Gerken J, Schweer T, Yarza P, Peplies J, and Glockner FO. 2013. The SILVA ribosomal RNA gene database project: improved data processing and web-based tools. Nucleic Acids Research 41:D590-596. 10.1093/nar/gks1219

Rognes T, Flouri T, Nichols B, Quince C, and Mahé F. 2016. VSEARCH: a versatile open source tool for metagenomics. PeerJ 4:e2584.

Roper M, Ellison C, Taylor JW, and Glass NL. 2011. Nuclear and genome dynamics in multinucleate ascomycete fungi. Current Biology 21:R786-793. 10.1016/j.cub.2011.06.042

Schloss PD, Westcott SL, Ryabin T, Hall JR, Hartmann M, Hollister EB, Lesniewski RA, Oakley BB, Parks DH, Robinson CJ, Sahl JW, Stres B, Thallinger GG, Van Horn DJ, and Weber CF. 2009. Introducing mothur: open-source, platform-independent, communitysupported software for describing and comparing microbial communities. Applied and Environmental Microbiology 75:7537-7541. 10.1128/AEM.01541-09

Schnell IB, Bohmann K, and Gilbert MTP. 2015. Tag jumps illuminated-reducing sequence-to-sample misidentifications in metabarcoding studies. Molecular Ecology Resources 15:1289-1303.

Schoch CL, Robbertse B, Robert V, Vu D, Cardinali G, Irinyi L, Meyer W, Nilsson RH, Hughes K, Miller AN, Kirk PM, Abarenkov K, Aime MC, Ariyawansa HA, Bidartondo M, Boekhout T, Buyck B, Cai Q, Chen J, Crespo A, Crous PW, Damm U, De Beer ZW, Dentinger BTM, Divakar PK, Dueñas M, Feau N, Fliegerova K, García MA, Ge Z-W, Griffith GW, Groenewald JZ, Groenewald M, Grube M, Gryzenhout M, Gueidan C, Guo L, Hambleton

Peer] reviewing PDF | (2018:02:25077:1:1:NEW 14 May 2018) 
812

813

814

815

816

817

818

819

820

821

822

823

824

825

826

827

828

829

830

831

832

833

834

835

836

837

838

839

840

841

842

843

844

845

846

847

848

849

850

851

852

853

854

855

856

857

858

859

860

861

S, Hamelin R, Hansen K, Hofstetter V, Hong S-B, Houbraken J, Hyde KD, Inderbitzin P, Johnston PR, Karunarathna SC, Kõljalg U, Kovács GM, Kraichak E, Krizsan K, Kurtzman CP, Larsson K-H, Leavitt S, Letcher PM, Liimatainen K, Liu J-K, Lodge DJ, Luangsa-ard JJ, Lumbsch HT, Maharachchikumbura SSN, Manamgoda D, Martín MP, Minnis AM, Moncalvo J-M, Mulè G, Nakasone KK, Niskanen T, Olariaga I, Papp T, Petkovits T, Pino-Bodas R, Powell MJ, Raja HA, Redecker D, Sarmiento-Ramirez JM, Seifert KA, Shrestha B, Stenroos S, Stielow B, Suh S-O, Tanaka K, Tedersoo L, Telleria MT, Udayanga D, Untereiner WA, Diéguez Uribeondo J, Subbarao KV, Vágvölgyi C, Visagie C, Voigt K, Walker DM, Weir BS, Weiss M, Wijayawardene NN, Wingfield MJ, Xu JP, Yang ZL, Zhang N, Zhuang W-Y, and Federhen S. 2014. Finding needles in haystacks: linking scientific names, reference specimens and molecular data for Fungi. Database : the journal of biological databases and curation 2014.

10.1093/database/bau061

Schoch CL, Seifert KA, Huhndorf S, Robert V, Spouge JL, Levesque CA, Chen W, Bolchacova E, Voigt K, and Crous PW. 2012. Nuclear ribosomal internal transcribed spacer (ITS) region as a universal DNA barcode marker for Fungi. Proceedings of the National Academy of Sciences 109:6241-6246.

Simon UK, and Weiss M. 2008. Intragenomic variation of fungal ribosomal genes is higher than previously thought. Molecular Biology and Evolution 25:2251-2254. 10.1093/molbev/msn188

Smith DP, and Peay KG. 2014. Sequence depth, not PCR replication, improves ecological inference from next generation DNA sequencing. PloS One 9:e90234. 10.1371/journal.pone.0090234

Šošic M, and Šikic M. 2017. Edlib: a C/C ++ library for fast, exact sequence alignment using edit distance. Bioinformatics (Oxford, England) 33:1394-1395. 10.1093/bioinformatics/btw753

Taylor DL, Walters WA, Lennon NJ, Bochicchio J, Krohn A, Caporaso JG, and Pennanen T. 2016. Accurate estimation of fungal diversity and abundance through improved lineagespecific primers optimized for Illumina amplicon sequencing. Applied and Environmental Microbiology 82:7217-7226. 10.1128/AEM.02576-16

Tedersoo L, Tooming-Klunderud A, and Anslan S. 2018. PacBio metabarcoding of Fungi and other eukaryotes: errors, biases and perspectives. New Phytologist 217:1370-1385. 10.1111/nph.14776

Tonge DP, Pashley CH, and Gant TW. 2014. Amplicon-based metagenomic analysis of mixed fungal samples using proton release amplicon sequencing. PloS One 9:e93849. 10.1371/journal.pone.0093849

Tourlousse DM, Yoshiike S, Ohashi A, Matsukura S, Noda N, and Sekiguchi Y. 2017. Synthetic spike-in standards for high-throughput $16 \mathrm{~S}$ rRNA gene amplicon sequencing. Nucleic Acids Research 45:e23. 10.1093/nar/gkw984

van der Walt Sf, Colbert SC, and Varoquaux GI. 2011. The NumPy Array: A Structure for Efficient Numerical Computation. Computing in Science \& Engineering 13:22-30. 10.1109/MCSE.2011.37

Vesty A, Biswas K, Taylor MW, Gear K, and Douglas RG. 2017. Evaluating the impact of DNA extraction method on the representation of human oral bacterial and fungal communities. PloS One 12:e0169877. 10.1371/journal.pone.0169877

Wang Q, Garrity GM, Tiedje JM, and Cole JR. 2007. Naive Bayesian classifier for rapid assignment of rRNA sequences into the new bacterial taxonomy. Applied and Environmental Microbiology 73:5261-5267. 10.1128/AEM.00062-07

Wang X-C, Liu C, Huang L, Bengtsson-Palme J, Chen H, Zhang JH, Cai D, and Li JQ. 2015. ITS1: a DNA barcode better than ITS2 in eukaryotes? 
862 White T, Bruns TD, Lee S, and Taylor J. 1990. Amplification and direct sequencing of fungal

863

864

865

866

867

868

869 ribosomal RNA genes for phylogenetics. PCR protocols: a guide to methods and applications:315 - 322.

Wright ES, and Vetsigian KH. 2016. Quality filtering of Illumina index reads mitigates sample cross-talk. BMC Genomics 17:876. 10.1186/s12864-016-3217-x

Wu J-Y, Jiang X-T, Jiang Y-X, Lu S-Y, Zou F, and Zhou H-W. 2010. Effects of polymerase, template dilution and cycle number on PCR based $16 \mathrm{~S}$ rRNA diversity analysis using

870 the deep sequencing method. BMC Microbiology 10:255. 10.1186/1471-2180-10-255 


\section{Table $\mathbf{1}$ (on next page)}

Summary statistics of the fungal ITS molecular barcode in comparison to bacterial $16 \mathrm{~S}$. 
1 Table 1. Summary statistics of the fungal ITS molecular barcode in comparison to bacterial $16 \mathrm{~S}$.

\begin{tabular}{|lccccc|} 
Region & Num & Avg Length (bp) & $\% \mathrm{HP}^{1}>6$ & $\% \mathrm{HP}^{1}>8$ & $\%>450 \mathrm{bp}$ \\
& Seqs & & & & - \\
ITS Full Length & 696704 & 488 & $55.07 \%$ & $8.66 \%$ & $3.27 \%$ \\
ITS1 & 685399 & 247 & $36.58 \%$ & $5.60 \%$ & $0.83 \%$ \\
ITS2 & 535200 & 264 & $44.19 \%$ & $5.54 \%$ & - \\
16S (V3/V4) & 627247 & 253 & $23.74 \%$ & $1.02 \%$ & \\
\hline
\end{tabular}

2 HP: homopolymer stretches

3 
Table 2 (on next page)

Comparison of HTAS software pipelines on the Ion Torrent PGM sequencing platform. 
1 Table 2. Comparison of HTAS software pipelines on the lon Torrent PGM sequencing platform.

\begin{tabular}{lcccccccc} 
Pipeline & $\begin{array}{l}\text { Total } \\
\text { OTUs }\end{array}$ & $\begin{array}{c}\text { Reads in } \\
\text { OTU table }\end{array}$ & $\begin{array}{c}\text { SynMock } \\
\text { OTUs } \\
(\mathrm{n}=12)\end{array}$ & $\begin{array}{c}\text { SynMock } \\
\text { error } \\
\text { rate }\end{array}$ & $\begin{array}{c}\text { BioMock } \\
\text { OTUs } \\
(\mathrm{n}=23)\end{array}$ & $\begin{array}{c}\text { BioMock } \\
\text { error } \\
\text { rate }^{1}\end{array}$ & $\begin{array}{c}\text { Primer } / \\
\text { Adapter } \\
\text { contam. }^{2}\end{array}$ & $\begin{array}{c}\text { Run time } \\
(\mathrm{min})^{3}\end{array}$ \\
\hline $\begin{array}{l}\text { AMPtk } \\
\text { (UPARSE) }\end{array}$ & 901 & 1830315 & $30(12)$ & $0.099 \%$ & $30(23)$ & $0.057 \%$ & $0.00 \%$ & 17 \\
$\begin{array}{l}\text { USEARCH } \\
\text { (UPARSE) }\end{array}$ & 821 & 1823711 & $32(11)$ & $1.573 \%$ & $32(23)$ & $0.054 \%$ & $63.63 \%$ & 215 \\
$\begin{array}{l}\text { AMPtk } \\
\text { (DADA2) }\end{array}$ & 814 & 1822013 & $30(12)$ & $0.099 \%$ & $31(23)$ & $0.058 \%$ & $0.00 \%$ & 42 \\
$\begin{array}{l}\text { QIIME2 } \\
\text { (DADA2) }\end{array}$ & 277 & 676282 & $8(5)$ & $2.276 \%$ & $21(19)$ & $0.043 \%$ & $97.11 \%$ & 41 \\
\hline
\end{tabular}

2 Error rate is the percent of total mismatches / total number of nucleotides

3 'Primers and adapter contamination was measured by using the 'search_oligodb' method in 4 USEARCH v10.0.240.

$5{ }^{3}$ Data were run using 10 cpus/threads on a Mac Pro OS 10.13 .3 (12 core $2.4 \mathrm{GHz}$ Intex Xeon 6 processor with 64 GB of RAM) 


\section{Table 3 (on next page)}

Comparison of HTAS software pipelines on the Illumina MiSeq sequencing platform (2x300bp). 
1 Table 3. Comparison of HTAS software pipelines on the Illumina MiSeq sequencing platform 2 (2x300bp).

\begin{tabular}{lcccccccc} 
Pipeline & $\begin{array}{l}\text { Total } \\
\text { OTUs }\end{array}$ & $\begin{array}{c}\text { Reads in } \\
\text { OTU table }\end{array}$ & $\begin{array}{c}\text { SynMock } \\
\text { OTUs } \\
(\mathrm{n}=12)\end{array}$ & $\begin{array}{c}\text { SynMock } \\
\text { error } \\
\text { rate } 1\end{array}$ & $\begin{array}{c}\text { BioMock } \\
\text { OTUs } \\
(\mathrm{n}=23)\end{array}$ & $\begin{array}{c}\text { BioMock } \\
\text { error } \\
\text { rate }^{1}\end{array}$ & $\begin{array}{c}\text { Primer } / \\
\text { Adapter } \\
\text { contam. }^{2}\end{array}$ & $\begin{array}{c}\text { Run time } \\
(\mathrm{min})^{3}\end{array}$ \\
\hline $\begin{array}{l}\text { AMPtk } \\
\text { (UPARSE) }\end{array}$ & 2007 & 13636429 & $33(12)$ & $0.063 \%$ & $36(23)$ & $0.031 \%$ & $0.00 \%$ & 67 \\
$\begin{array}{l}\text { USEARCH } \\
\text { (UPARSE })\end{array}$ & 1924 & 12073312 & $43(11)$ & $1.047 \%$ & $33(23)$ & $0.029 \%$ & $0.00 \%$ & 55 \\
$\begin{array}{l}\text { AMPtk } \\
\text { (DADA2) }\end{array}$ & 1954 & 13628802 & $34(12)$ & $0.063 \%$ & $42(23)$ & $0.032 \%$ & $0.00 \%$ & 470 \\
$\begin{array}{l}\text { QIIME2 } \\
\text { (DADA2) }\end{array}$ & 786 & 8522714 & $16(10)$ & $1.186 \%$ & $29(22)$ & $0.901 \%$ & $53.43 \%$ & 269 \\
$\begin{array}{l}\text { PIPITS } \\
\text { (ITSx) }\end{array}$ & 2784 & 9798280 & $20(0)$ & $100 \%$ & $48(22)$ & $0.744 \%$ & $0.00 \%$ & 1108 \\
\hline
\end{tabular}

$3{ }^{1}$ Error rate is the percent of total mismatches / total number of nucleotides

4 'Primers and adapter contamination was measured by using the 'search_oligodb' method in 5 USEARCH v10.0.240.

6 3Data were run using 10 cpus/threads on a Mac Pro OS 10.13 .3 (12 core $2.4 \mathrm{GHz}$ Intex Xeon 7 processor with $64 \mathrm{~GB}$ of RAM)

$8{ }^{4}$ VSEARCH was used for dereplication (finding unique sequences), quality filtering, and

9 constructing OTU table due to data being too large for 32-bit USEARCH10. 


\section{Figure 1}

The fungal internal transcribed spacer (ITS) region of the rRNA array is highly variable in length.

(A) A schematic of the rRNA array highlights the conserved priming sites commonly used to amplify either the ITS1 or ITS2 region. (B) Size distribution of full length ITS (blue), ITS1 (green), ITS2 (red) sequences in the UNITE v7.2 curated databases shown in comparison to the bacterial 16S V3/V4 amplicon from the Silva v128 database. Current sequencing technologies do not have read lengths long enough to capture full-length ITS sequences, and thus ITS1 or ITS2 regions are used for fungal environmental community analysis. 16S V3/V4 in yellow; ITS full length in blue, ITS2 in red, and ITS1 in green. 

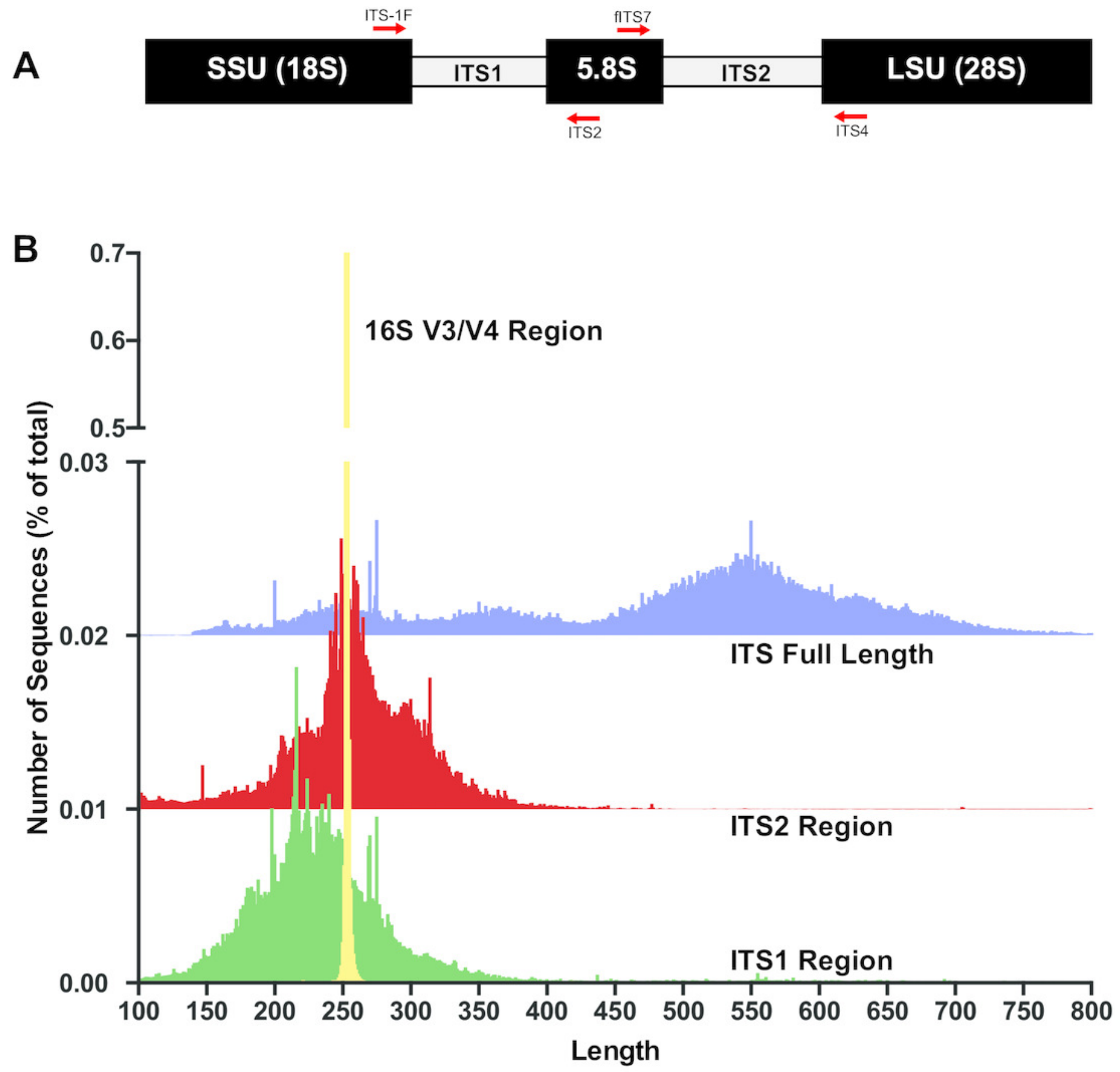


\section{Figure 2}

Pre-processing ITS sequences is critically important to accurately recover OTUs using the curated UNITE v7.2 reference database.

ITS1 and ITS2 sequences were extracted from the UNITE v7.2 general fasta release database using 'AMPtk database'. Identical sequences were collapsed (dereplication) and remaining sequences were clustering using UPARSE ('cluster_otus) to generate the total number of UPARSE OTUs expected for the (A) ITS1 and (B) ITS2 regions. The data was then processed to five different lengths $(150,200,250,300$, and $350 \mathrm{bp})$ and then clustered (UPARSE 'cluster_otus') using i) default UPARSE truncation (longer sequences are truncated and shorter sequences are discarded), ii) padding with ambiguous bases (longer sequences truncated and shorter sequences padded with N's to length threshold), and iii) full-length sequences (longer sequences are truncated and shorter sequences are retained if reverse primer is found). Full-length and padding pre-processing sequences outperforms default UPARSE truncation. 


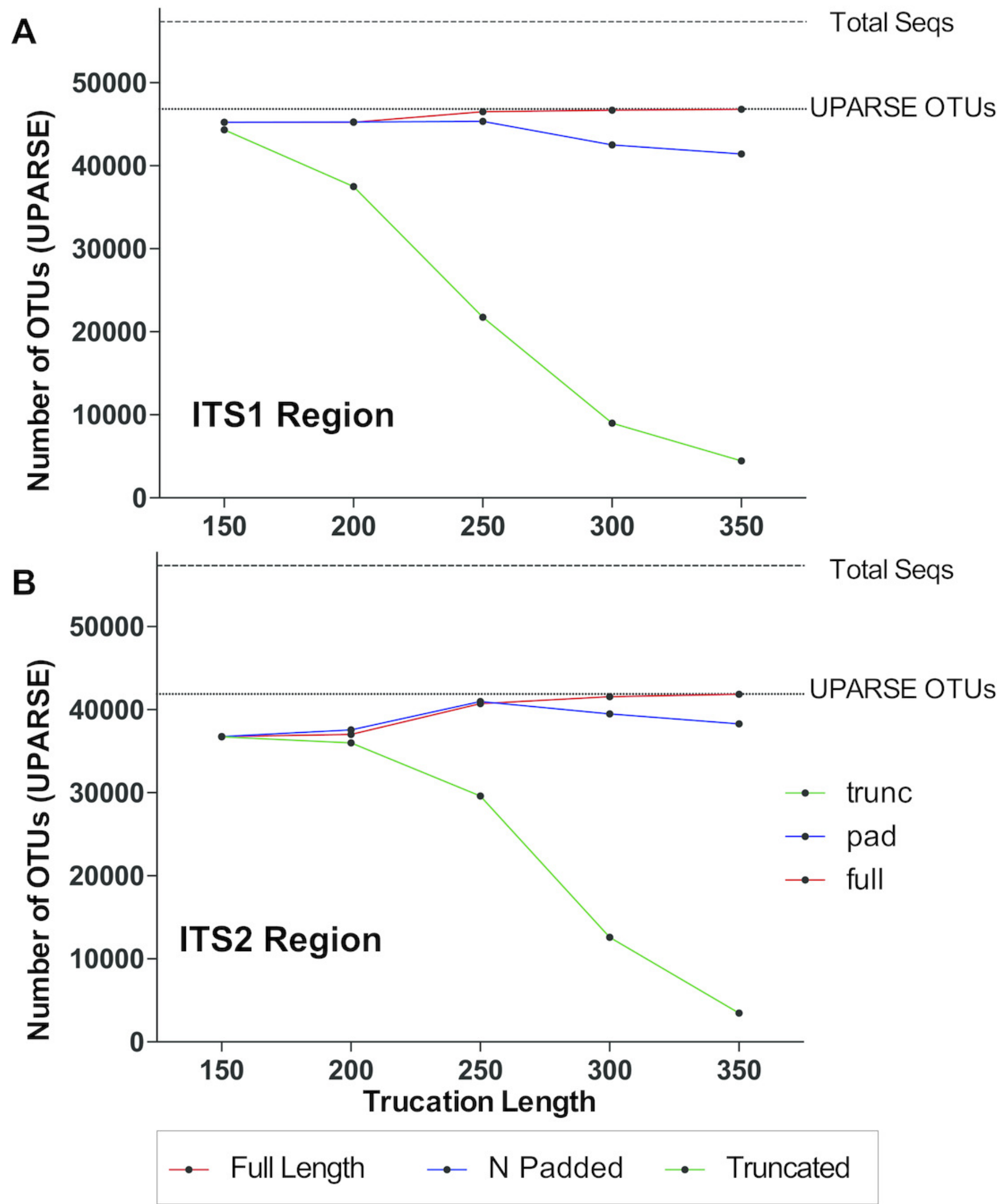


Figure 3

Overview of the commands in AMPtk.

AMPtk is built to be compatible with multiple sequencing platforms as well as contains several clustering algorithms. 
1. Find barcode (Ion /454), relabel header

2. Find/Trim Forward and Reverse Primers

3. Merge PE reads (Illumina only)

4. Trim/Pad read to set length (optional)

5. Combine samples (IIlumina only)

6. Create sample mapping file (QIIME-like map file)

7. Quality filter reads (expected errors filtering)

8. Run "Clustering"

- UPARSE: 97\% clustering into OTUs

- DADA2: de-noising into inferred sequences

- UNOISE2/3: de-noising into inferred sequences

9. Reference chimera filtering (optional)

10. Map reads to OTUs and/or ASVs

11. Create OTU table

12. Map OTUs/ASVs to mock community (optional)

13. Calculate index-switching rate between samples

14. Index-switching filter, remove counts below rate

15. Update FASTA OTUs/ASVs and OTU table

16. Pre-formatted databases for ITS, 16S, COI, and LSU can be downloaded with 'amptk install'

17. Assign taxonomy using "hybrid" approach

- Global alignment to reference DB

- UTAX Classifier based on trained DB

- SINTAX Classifier based on reference DB

- parse results and choose best taxonomy

18. Ouptut taxonomy, append to OTU table, create BIOM

\section{amptk SRA-submit}

amptk heatmap 


\section{Figure 4}

Read abundance is an unreliable proxy for actual abundance within a mixed community.

Using an equimolar mixture of cloned ITS sequences in plasmid form (MockA, MockB1, MockB2) in comparison to equimolar mixture of individual PCR products (Stds) illustrates that the initial PCR reaction during library preparation heavily biases the read abundance obtained after sequencing on both the Ion Torrent PGM and Illumina MiSeq platforms. While read abundances are unreliable, all members of the mock community were recovered. MockA represents a 1:16,000 dilution and MockB1/MockB2 are replicates of a 1:32,000 dilution of the BioMock community. The lon Torrent PGM platform has a length threshold of approximately $450 \mathrm{bp}$; therefore longer amplicons like Wolfiporia cocos ITS2 sequence very poorly. 


\begin{tabular}{|c|c|c|c|c|c|c|c|c|c|c|c|c|}
\hline \multirow[b]{2}{*}{ Species } & \multirow[b]{2}{*}{$\begin{array}{l}\text { ITS2 } \\
\text { Length }\end{array}$} & \multirow[b]{2}{*}{ Content } & \multirow[b]{2}{*}{$H P>5$} & \multirow[b]{2}{*}{ ID } & \multicolumn{4}{|c|}{ Ion Torrent PGM } & \multicolumn{4}{|c|}{ Illumina MiSeq } \\
\hline & & & & & $\frac{\infty}{0}$ & $\begin{array}{l}\varangle \\
\text { ㅇ } \\
\sum\end{array}$ & $\begin{array}{l}\bar{m} \\
\text { 음 } \\
\dot{\Sigma}\end{array}$ & 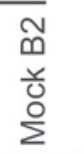 & $\frac{n}{0}$ & $\begin{array}{l}\frac{1}{0} \\
\text { ㅇ } \\
\sum\end{array}$ & $\begin{array}{l}\bar{n} \\
\text { ㅇ } \\
\dot{\Sigma}\end{array}$ & 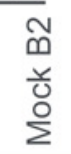 \\
\hline Phialocephala fusca & 237 & $68.4 \%$ & 0 & mock1 & 4905 & 19 & 6 & 1 & 8615 & 725 & 329 & 3337 \\
\hline Ascomycete sp. & 238 & $50.8 \%$ & 0 & mock2 & 5106 & 11651 & 10809 & 11877 & 9174 & 20763 & 26129 & 18341 \\
\hline Phialocephala lagerbergii & 238 & $58.8 \%$ & 0 & mock3 & 4886 & 13479 & 12111 & 13392 & 8648 & 28515 & 29482 & 21269 \\
\hline Helotiales sp. & 239 & $57.3 \%$ & 0 & mock4 & 4233 & 15219 & 13048 & 14896 & 9050 & 27726 & 32576 & 24276 \\
\hline Aspergillus candidus & 260 & $65.8 \%$ & 3 & mock5 & 2813 & 31 & 23 & 3 & 8992 & 147 & 122 & 269 \\
\hline Bjerkandera adusta & 281 & $51.2 \%$ & 0 & mock6 & 3977 & 8112 & 7172 & 7787 & 13597 & 13112 & 13866 & 15067 \\
\hline Laetiporus caribensis & 283 & $52.7 \%$ & 0 & mock7 & 3330 & 7810 & 6457 & 6365 & 9404 & 15035 & 16622 & 16385 \\
\hline Trametes gibbosa & 288 & $50.0 \%$ & 1 & mock8 & 3637 & 7281 & 6914 & 6865 & 8137 & 13819 & 14579 & 14787 \\
\hline Laetiporus gilbertsonii & 290 & $54.1 \%$ & 0 & mock9 & 4066 & 8831 & 10401 & 12638 & 8751 & 22860 & 21680 & 20682 \\
\hline Gloeoporus pannocinctus & 292 & $43.8 \%$ & 0 & mock10 & 2603 & 2922 & 3025 & 2567 & 9718 & 11150 & 11792 & 14265 \\
\hline Wolfiporia dilatohypha & 293 & $54.6 \%$ & 0 & mock11 & 3957 & 94 & 110 & 109 & 8775 & 243 & 224 & 194 \\
\hline Schizopora sp. & 293 & $48.1 \%$ & 0 & mock12 & 4037 & 6965 & 7030 & 6626 & 8676 & 12857 & 13947 & 14860 \\
\hline Fomitopsis ochracea & 295 & $44.1 \%$ & 0 & mock13 & 3689 & 2913 & 2860 & 2651 & 9471 & 5522 & 5432 & 6883 \\
\hline Laetiporus cremeioporus & 296 & $54.7 \%$ & 0 & mock14 & 3922 & 10279 & 11920 & 12440 & 8262 & 16454 & 16390 & 16798 \\
\hline Phanerochaete laevis & 300 & $47.7 \%$ & 1 & mock15 & 3863 & 6970 & 7650 & 6876 & 9242 & 15667 & 15543 & 18168 \\
\hline Laetiporus cincinnatus & 302 & $54.0 \%$ & 0 & mock16 & 3133 & 5699 & 7645 & 7505 & 7675 & 16819 & 16157 & 14608 \\
\hline Punctularia strigosozonata & 303 & $53.1 \%$ & 0 & mock17 & 4019 & 8271 & 7688 & 8217 & 7669 & 10701 & 11572 & 11671 \\
\hline Phellinus cinereus & 314 & $49.7 \%$ & 0 & mock18 & 3672 & 2937 & 2985 & 2597 & 9807 & 6314 & 5953 & 7496 \\
\hline Antrodiella semisupina & 315 & $43.8 \%$ & 1 & mock19 & 3089 & 3047 & 3406 & 2741 & 9297 & 9356 & 8990 & 11593 \\
\hline Leptoporus mollis & 315 & $45.4 \%$ & 3 & mock20 & 3551 & 4969 & 4320 & 4028 & 9047 & 8847 & 8747 & 9987 \\
\hline Leptoporus mollis 2 & 315 & $45.1 \%$ & 1 & mock21 & 3776 & 207 & 366 & 249 & 9250 & 405 & 302 & 414 \\
\hline Mortierellales sp. & 353 & $45.0 \%$ & 0 & mock22 & 3264 & 4668 & 4311 & 3812 & 9151 & 10865 & 9728 & 13365 \\
\hline Laetiporus persicinus & 379 & $51.2 \%$ & 2 & mock23 & 2147 & 2651 & 2385 & 2053 & 6486 & 488 & 421 & 521 \\
\hline Penicillium nothofagi & 260 & $66.2 \%$ & 1 & mock24 & 3644 & NA & NA & NA & 8278 & NA & NA & NA \\
\hline Metapochonia suchlasporia & 291 & $64.6 \%$ & 1 & mock25 & 1976 & $\mathrm{NA}$ & NA & NA & 2045 & NA & NA & NA \\
\hline Wolfiporia cocos & 548 & $59.7 \%$ & 0 & mock26 & 7 & NA & NA & NA & 5979 & NA & NA & NA \\
\hline
\end{tabular}




\section{Figure 5}

Schematic drawing of the 12-member non-biological synthetic mock community (SynMock).

Conserved priming sites for either ITS1 or ITS2 amplicons are retained for versatility. The length distribution, GC content, and homopolymer stretches are representative of curated public databases; however, the sequences are non-biological and thus not found in nature.

ITS1

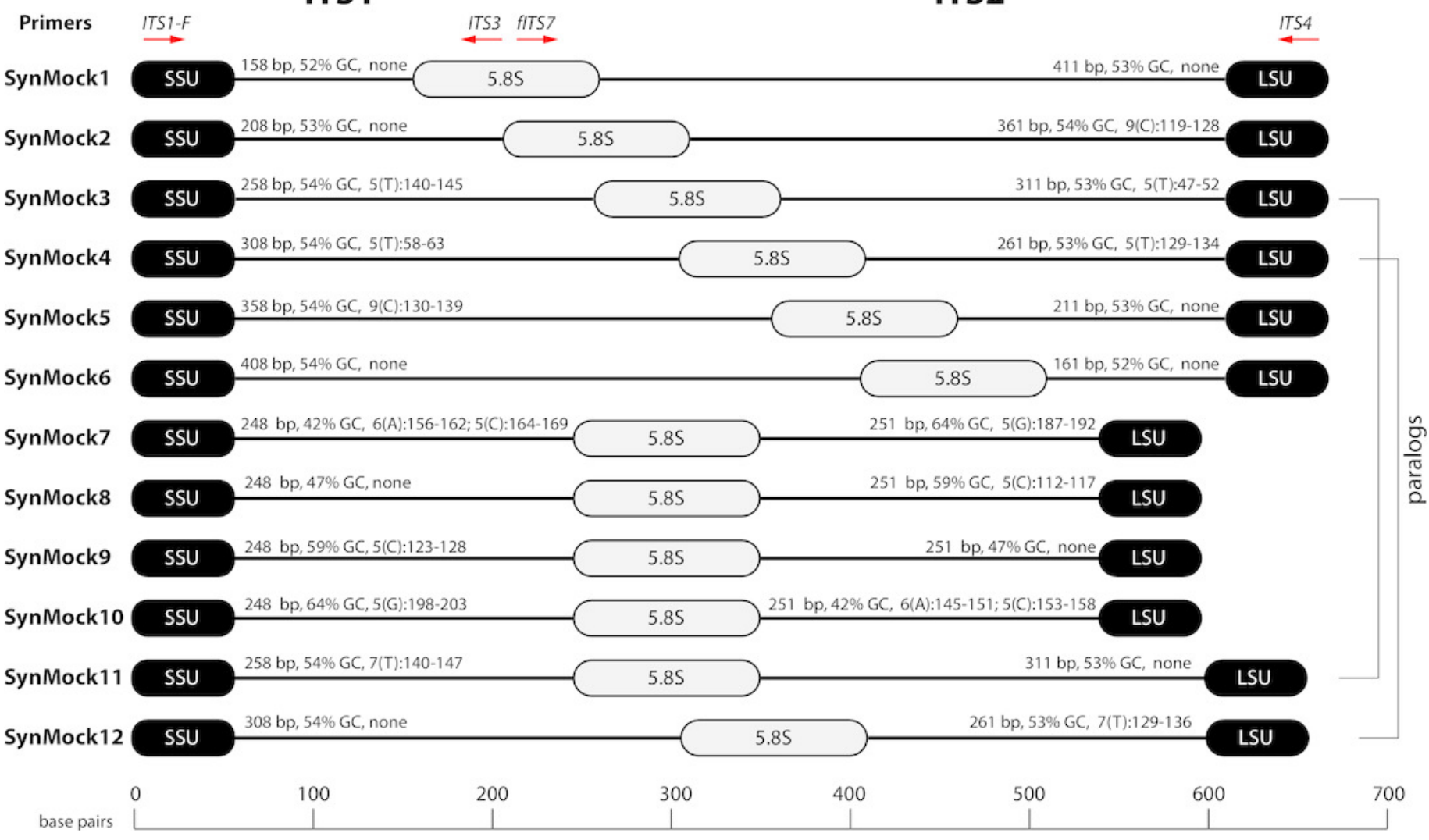




\section{Figure 6}

Index-bleed or sample mis-assignment occurs on both Ion Torrent PGM and Illumina Miseq.

(A) Read counts from the SynMock community run on the Ion Torrent PGM platform. SynMock reads can be found in environmental samples and reads from the environmental samples are found in the SynMock sample. The data were processed allowing 0 mismatches in the barcode sequence and there is no clear pattern to index-bleed on the lon Torrent PGM platform. (B) Data processed on the Illumina MiSeq $(2 \times 300)$ allowing 0 mismatches in the index reads show index-bleed in and out of the SynMock sample. Samples that share an index (i5 or i7) show an increase in index-bleed. (C) Index-bleed between samples can be tracked using the SynMock spike-in control, where AMPtk will measure both index-bleed into the SynMock as well as index-bleed into other samples. These calculated values are then used by AMPtk to filter an OTU table to remove read counts that fall below the index-bleed threshold. Index-bleed is reduced if 0 mismatches are allowed in the barcode/index sequence, however, this is still not sufficient to eliminate index-bleed. 


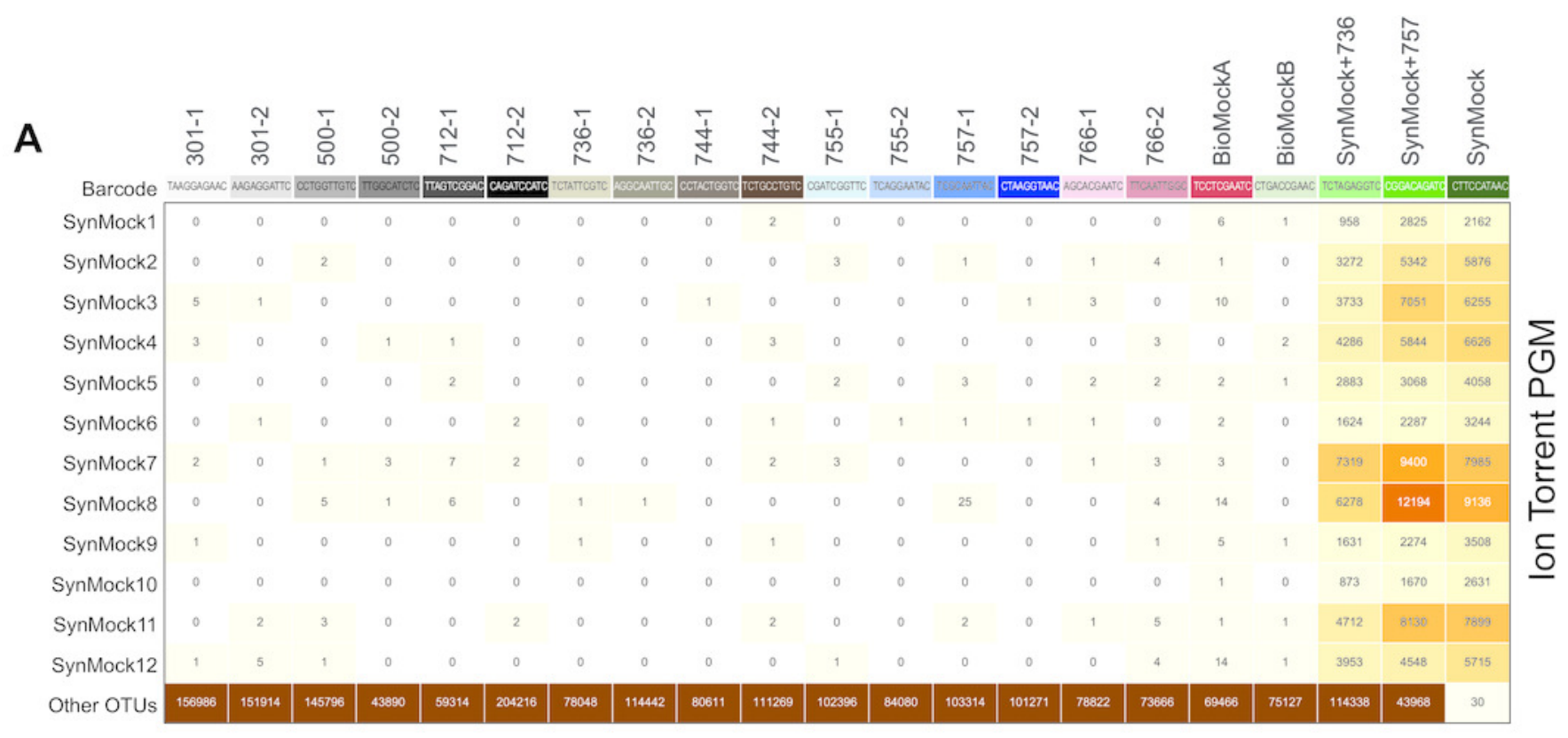

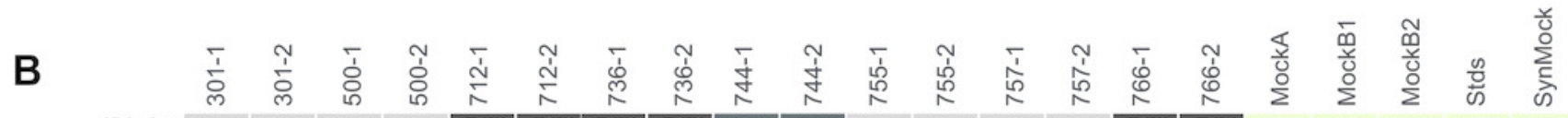

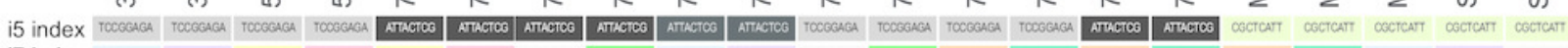

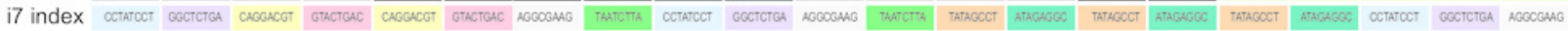
\begin{tabular}{l|llllllllllllllllllllll} 
SynMock1 & 0 & 0 & 0 & 0 & 0 & 0 & 8 & 0 & 0 & 0 & 5 & 0 & 0 & 0 & 0 & 0 & 0 & 0 & 0 & 0 & 71892 \\
\hline
\end{tabular}

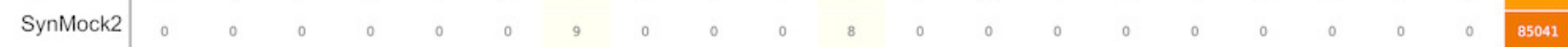

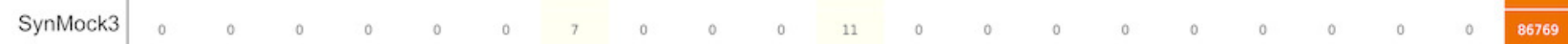

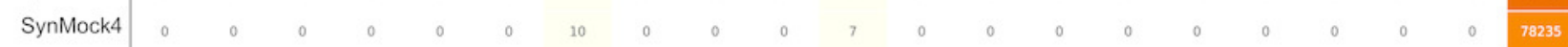
\begin{tabular}{c|cccccccccccccccccccccccc} 
SynMock5 & 0 & 0 & 0 & 0 & 0 & 0 & 13 & 0 & 0 & 0 & 22 & 0 & 0 & 0 & 0 & 0 & 0 & 0 & 0 & 94680
\end{tabular} \begin{tabular}{l|lllllllllllllllllllllll} 
SynMock6 & 0 & 0 & 0 & 0 & 0 & 0 & 14 & 0 & 0 & 0 & 8 & 0 & 0 & 0 & 0 & 0 & 0 & 0 & 0 & 0 & 72994
\end{tabular} \begin{tabular}{l|lllllllllllllllllllll} 
SynMock7 & 0 & 0 & 0 & 0 & 0 & 0 & 13 & 0 & 0 & 0 & 16 & 0 & 0 & 0 & 0 & 0 & 0 & 0 & 0 & 0 & 103824 \\
\hline
\end{tabular}

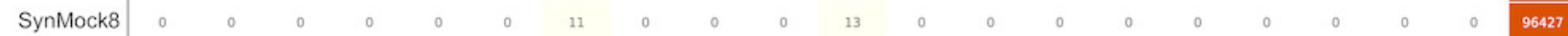
SynMock9 SynMock10 SynMock11 SynMock12

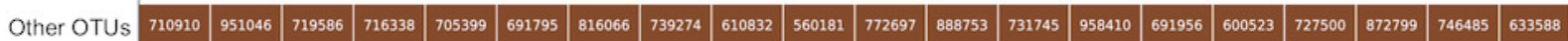

C

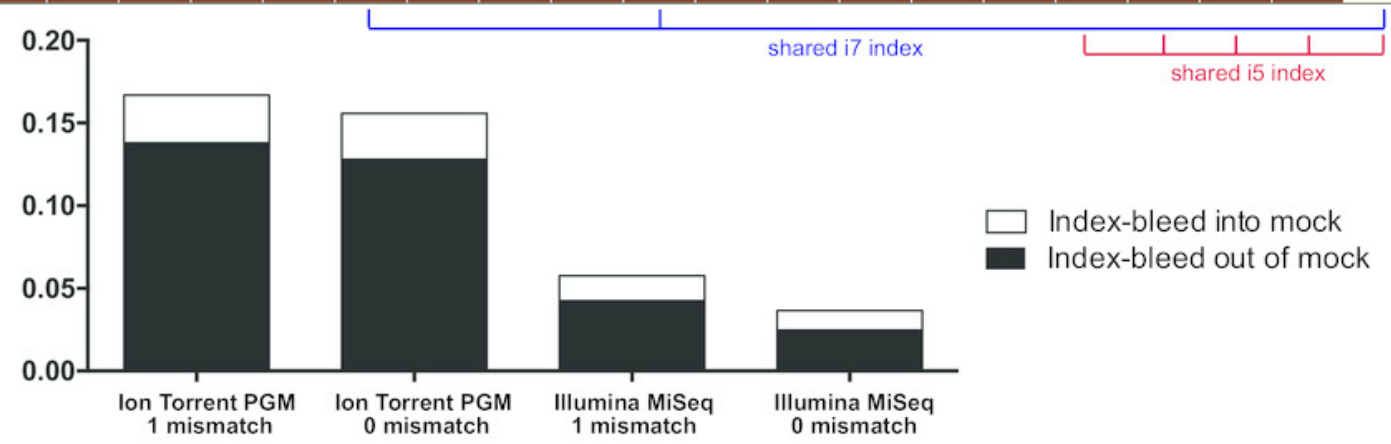

